\title{
On the counting tensor model observables as $U(N)$ and $O(N)$ classical invariants
}

\author{
Joseph Ben Geloun* \\ LIPN, UMR CNRS 7030, Université Sorbonne Paris Nord, 93430 Villetaneuse, France \\ International Chair in Mathematical Physics and Applications \\ ICMPA-UNESCO Chair, 072Bp50, Cotonou, Benin \\ E-mail: bengeloun@lipn.univ-paris13.fr
}

\begin{abstract}
Real or complex tensor model observables, the backbone of the tensor theory space, are classical (unitary, orthogonal, symplectic) Lie group invariants. These observables represent as colored graphs, and that representation gives an handle to study their combinatorial, topological and algebraic properties. We give here an overview of the symmetric group-theoretic formulation of the enumeration of unitary and orthogonal invariant observables which turns out to bear a rich structure. From their counting formulae, one finds a correspondence with topological field theory on 2-cellular complexes that brings other interpretations of the same countings. Furthermore, tensor model observables span an algebra that turns out to be semi-simple. Dealing with complex tensors, we discuss the representation theoretic base of the algebra making explicit its Wedderburn-Artin decomposition. The real case is more subtle as a base of its Wedderburn-Artin decomposition is yet unknown.
\end{abstract}

Corfu Summer Institute 2019 "School and Workshops on Elementary Particle Physics and Gravity" (CORFU2019)

31 August - 25 September 2019

Corfù, Greece

${ }^{*}$ Speaker. 


\section{Introduction}

In any physical model, interactions and observables play a fundamental role. For tensor models [1]-[5], these observables build from the contractions of multidimensional arrays or tensors that transform covariantly under the action of some classical Lie groups. The most recent studies on tensor models over Lie groups consider $U(N)$, the unitary group of order $N$, and $O(N)$, the orthogonal group of order $N$. Note that much less is known about tensor models with $\operatorname{Sp}(2 N)$-invariants, $S p(2 N)$ being the (real or complex) symplectic group, see [6] and [7]. Defined by contractions of tensors, the observables or interactions of tensor models simply become polynomial invariants of these classical Lie groups (for short we shall call them tensor invariants). Correlators in such models compute therefore, at the perturbative level, in terms of Feynman graphs involving tensor invariants as their vertices. We can easily foresee that the quantum field theory calculations heavily rely on the diagrammatics and combinatorics of these objects. Hence, a systematic combinatorial study of $U(N)$ and $O(N)$ classical invariants has been launched in the recent years bringing already a wealth of core results $[8,9,10,11,12,13,14,15,16,7,17,18]$

A preferred way of enumerating these invariants mainly rests on algebraic techniques of the symmetric groups. There are a lot of reasons why the use symmetric groups has become a natural reflex and a dominating tool in the combinatorial study of tensor invariants. Indeed, the success of such studies has strongly benefited from the expertise and techniques developed increasingly for matrices in recent years. Matrix models interconnect, in a nonexhaustive fashion, integrable models, 2D gravity, gauge theory, string theory and Riemannian geometry. The symmetric groups and their representation were established master tools to tame correlators and observables of matrix models, and thereby to understand the half-BPS sector of $\mathscr{N}=4$ SYM [19, 20, 21, 22]. This success emanates from importing Schur-Weyl duality as an instrument for grasping Gauge-String duality [23]. Furthermore, this algebraic implement highlights new correspondences between countings in quantum field theory, matrix models, and string theory [24, 25, 26, 27, 28, 29, 30]. With all the results on matrix correlators, it may come as no surprise that a similar approach extends to tensor models and yields applications even beyond the realm of theoretical physics. In quantum information processing [31] and linguistics [32][33] one also finds that matrix models and symmetric groups gather a renewed attention.

A few words about the interest for tensor models fall in line. Tensor models [1, 2, 3] were introduced as candidate theories for quantum gravity in higher dimensions [35, 34, 36]. As expected, they were much more difficult to address than matrix models [4], one of the few successful candidate approaches quantizing gravity in 2D. The theory of tensors became only tractable after the inception of colored tensors [37]. This class of tensor models supports a large $N$ expansion [38] in a similar way that matrix models expand by the famous ' $t$ Hooft large $N$ limit [39]. After, a variety of results came to light: the critical behavior of tensor models was uncovered analytically [40, 41, 42, 43], entire new families of quantum tensor field theories were found renormalizable both at the perturbative [45]-[53] and at the non-perturbative levels [54]-[63]. More recently, the Sachdev-Ye-Kitaev (SYK) condensed matter model $[64,65]$ proves to have the same diagrammatics as colored tensor models at large $N$ [66]. This opens a whole new avenue of research giving rise to an unprecedented interest of the community for tensor models (see [67]-[72] and references therein), hence the urge of finding new tools to understand them better. 
The manipulation of symmetric groups and its representation theory in tensor models has shed a new light on calculations, allowed one to discover genuine effects, and bridged theories by uncovering new correspondences (bijections between different-looking objects). It also reveals hidden structures at the interface of three domains: combinatorics, algebra and topology/geometry. The exact enumeration complex tensor invariants connects to a topological field theory (TopFT) that gives in return a geometrical interpretation of each observable as a branched cover of the 2-dimensional sphere [8]. Tensor invariants may be regarded as the generators of an algebra of observables with interesting properties (semi-simplicity, orthogonal bases, gradation) [11]. The two-point correlators of complex observables expand in these orthogonal bases. Many of these features of complex tensor models extend to the real case [7]. Once the enumeration of real tensor invariants sorted, their TopFT formulation finds a bijection with the covers of the torus with defects, and their algebra possesses also orthogonal bases and is semi-simple. As another interesting by product of these analyses, new integer sequences has been recorded in OEIS [73] while also some sequences therein got simplified.

This work delivers a summary of three contributions [8], [11], and [7]. We put in parallel the counting of $U(N)$ tensor invariants and its corollaries [8] [11] and that of $O(N)$ tensor invariants [7]. The next section introduces our notation. Then section 3 focuses on the counting of complex tensor osbervables. The following section 4 undertakes the same analysis but for real tensors before a conclusion is drawn in section 5 with some perspectives of this work.

\section{Notation: complex and real tensors}

The building blocks of the theory are complex and real tensors that we now introduce. This section follows $[8,7,5]$.

Consider a field $\mathbb{F}=\mathbb{R}, \mathbb{C}$, and $d$ vector spaces $V_{a}$ over $\mathbb{F}, a=1, \ldots, d, d \geq 2$, of respective dimensions $N_{a}$. We denote $\mathscr{U}(N)$ either $U(N)$ or $O(N)$ depending on the complex or real nature of the field $\mathbb{F}$, respectively. Let $T$ be a multilinear map $\otimes_{a=1}^{d} V_{a} \rightarrow \mathbb{F}$ that we call a tensor of rank $d$ with components $T_{p_{1}, \cdots, p_{d}} \in \mathbb{F}$ (also called, by abuse, the tensor itself), $p_{a}=1, \ldots, N_{a}$. As the group $\otimes_{a=1}^{d} \mathscr{U}\left(N_{a}\right)$ acts on $\otimes_{a=1}^{d} V_{a}$ in the fundamental representation, $T$ transforms under the tensor product of $d$ fundamental representations of the groups $\mathscr{U}\left(N_{a}\right)$. Each group $\mathscr{U}\left(N_{a}\right)$ acts independently on a tensor index $p_{a}$ and we write the component of the transformed tensor as:

$$
T_{p_{1}, \ldots, p_{d}}^{R}=\sum_{q_{k}} R_{p_{1} q_{1}}^{(1)} \ldots R_{p_{d} q_{d}}^{(d)} T_{q_{1}, \ldots, q_{d}}, \quad R^{(a)} \in \mathscr{U}\left(N_{a}\right) .
$$

The observables of real or complex tensor models are the contractions of the tensors $T_{p_{1}, \ldots, p_{d}}$ with respect to a trivial metric (however, the metric will be not trivial when dealing with the symplectic group, this case is not treated here; the interested reader is referred to [7]). Such contractions are invariant under the action of $\otimes_{a=1}^{d} \mathscr{U}\left(N_{a}\right)$ (note that there is subtlety in the complex case as the action of $R \in \mathscr{U}\left(N_{a}\right)$ on a $T$ entails the action of $R^{\dagger} \in \mathscr{U}\left(N_{a}\right)$ on a conjugate $\left.\bar{T}\right)$. One should pay attention on the fact that if we call these contractions $\mathscr{U}(N)$ ) invariants without making precise how many tensorial factors are involved, this is also an abuse. For real tensors, we must contract an even number of them to obtain an invariant. For complex tensors, only contractions from conjugate tensor components, $T$ and $\bar{T}$, are allowed. We can easily see that tensor contractions generalize 
matrix traces that are $\mathscr{U}(N)$ invariants: $\operatorname{Tr}\left(\left(M M^{\dagger}\right)^{n}\right), n \geq 1$, for a matrice $M$ of size $N$. For this reason, we use the same notation $\operatorname{Tr}(\cdot)$ for tensors, hereafter. Another important feature of tensor contractions is that they encode as $d$-regular graphs with edge coloring with $d$ different colors, and each color at every vertex (representing each tensor) represents an index of this tensor [37, 40]. We will come back on this property in the following section. Calling $\mathbf{b}$ that representative colored graph, we denote the invariant equivalently by

$$
\begin{aligned}
\text { complex }: & O_{\mathbf{b}}(T, \bar{T}) & =\operatorname{Tr}_{\mathbf{b}}(\bar{T} \cdot T \ldots \bar{T} \cdot T) \\
\text { real : } & O_{\mathbf{b}}(T) & =\operatorname{Tr}_{\mathbf{b}}(T \cdot T \cdots T) .
\end{aligned}
$$

The dot means that some indices of the tensors get sum by other indices of other tensors. The data of the graph $\mathbf{b}$ is sufficient to determine according to which contraction pattern the tensor indices are summed, and to each tensor contraction we have a unique graph associated with it (up to isomorphism). The simplest non trivial tensor contraction denotes $\operatorname{Tr}_{2}(T, \bar{T})$ in the complex case and $\operatorname{Tr}_{2}(T)$ in the real case. They express as

$$
\begin{aligned}
\text { complex : } & \operatorname{Tr}_{2}(T, \bar{T}) & =\sum_{p_{k}=1, \ldots, N_{k}} T_{p_{1} p_{2} \ldots p_{d}} \bar{T}_{p_{1} p_{2} \ldots p_{d}} \\
\text { real : } & \operatorname{Tr}_{2}(T) & =\sum_{p_{k}=1, \ldots, N_{k}}\left(T_{p_{1} p_{2} \ldots p_{d}}\right)^{2} .
\end{aligned}
$$

This simple quadratic invariant in the components of the tensor will play an important role when we will define the Gaussian tensor field measure.

In the following, most of the illustrations are made at fixed rank $d=3$ but it generally extends in any $d$. We will also mostly reduce to $N_{a}=N$ for simplicity.

Examples of unitary invariants. Figure 1 illustrates contraction patterns for $U(N)^{\otimes 3}$ (complex) tensor invariants with 2 (one $T$ and one $\bar{T}$ ), 4 , and 6 complex tensors. They are bipartite and colored graphs. $\bar{T}$ is represented by a black vertex, $T$ by a white one. The color $a=1,2, \ldots, d$ of the edge is associated with the label $a$ of an index $p_{a}$ of the tensor. Note that the graphs are bipartite.
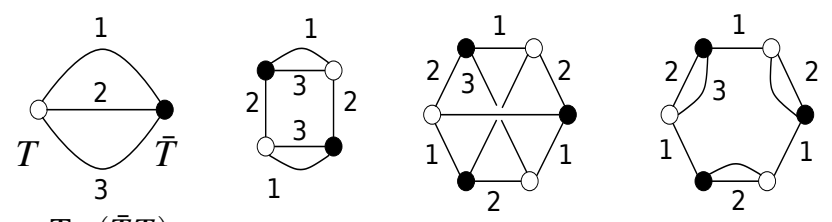

$$
\operatorname{Tr}_{2}(\bar{T} T)
$$

Figure 1: $U(N)^{\otimes 3}$ tensor invariants.

Examples of orthogonal invariants. Examples of $O(N)^{\otimes 3}$ tensor invariants are provided in Figure 2.

Tensor field measure. Tensor contractions define the interactions of tensor models. We introduce a physical model through a partition function (using abusively a single variable $T$ for both real and complex cases)

$$
Z=\int d v(T) \exp (-S(T))
$$



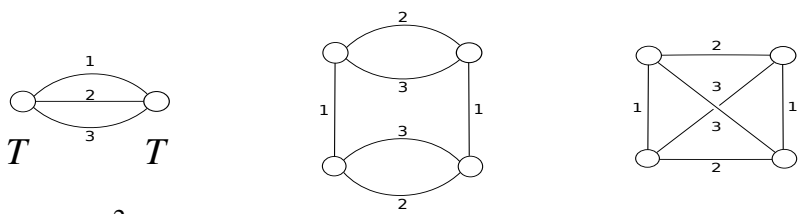

$\operatorname{Tr}_{2}\left(T^{2}\right)$

Figure 2: $O(N)^{\otimes 3}$ tensor invariants.

where $S(T)=\sum_{\mathbf{b}} \lambda_{\mathbf{b}} O_{\mathbf{b}}(T)$ is a finite sum over some $\mathscr{U}(N)$ invariants representing the model interactions, each associated with a coupling $\lambda_{\mathbf{b}}$. The Gaussian tensor field measure $d v(T)$ is given by

$$
\begin{array}{rlrl}
\text { complex : } & d v(T) & \equiv \prod_{i_{k}} d T_{i_{1} i_{2} \ldots i_{d}} d \bar{T}_{i_{1} i_{2} \ldots i_{d}} e^{-\operatorname{Tr}_{2}(T, \bar{T})} \\
\text { real : } & d v(T) \equiv \prod_{i_{k}} d T_{i_{1} i_{2} \ldots i_{d}} e^{-\operatorname{Tr}_{2}(T)}
\end{array}
$$

with $\operatorname{Tr}_{2}(T, \bar{T})$ and $\operatorname{Tr}_{2}(T)$ given by (2.3). We will be interested in the moment of these measures called the correlators of the models:

$$
\left\langle O_{\mathbf{b}}(T)\right\rangle=\frac{1}{Z_{0}} \int d v(T) O_{\mathbf{b}}(T) .
$$

with $Z_{0}=\int d v(T)$. The free propagator of the Gaussian measure corresponds to

$$
\left\langle T_{i_{1} i_{2} \ldots i_{d}} T_{j_{1} j_{2} \ldots j_{d}}\right\rangle=\frac{1}{Z_{0}} \int d v(T) T_{i_{1} i_{2} \ldots i_{d}} T_{j_{1} j_{2} \ldots j_{d}}=\delta_{i_{1} j_{1}} \delta_{i_{2} j_{2}} \ldots \delta_{i_{d} j_{d}} .
$$

We will also discuss the mean values of two observables, called 2pt-function,

$$
\left\langle O_{\mathbf{b}}(T) O_{\mathbf{b}^{\prime}}(T)\right\rangle=\frac{1}{Z_{0}} \int d v(T) O_{b}(T) O_{b^{\prime}}(T) .
$$

The second correlator will be restricted to normal order allowing only Wick contractions from $O_{\mathbf{b}}(T)$ to $O_{\mathbf{b}^{\prime}}(T)$. Using the symmetric group formulation of the $\mathscr{U}(N)$ invariants, we will reformulate (2.8) and analyse the representation algebraic structure brought by the 2 pt-function.

\section{Counting complex tensor model observables}

Symmetric groups offer an elegant formulation of the counting of invariants based on the contractions of $n$ copies of tensors $T_{i_{1}, \cdots, i_{d}}$ and $n$ copies of the conjugate tensors $\bar{T}_{i_{1}, \cdots, i_{d}}$. This enumeration problem addresses as a TopFT with permutation gauge group that we also discuss. Finally, translating the same counting in representation theory, we find a sum of terms involving the famous Kronecker coefficient. This section summarizes [8] and [11].

\subsection{Counting}

Counting complex tensor invariants performs with the aid of a graphical representation that we shall not refrain to explain. Each tensor $T_{p_{1}, \cdots, p_{d}}$ will be associated with a white vertex with $d$ exiting half-lines, each of which is representing an index $p_{a}, a=1, \ldots, d$. The conjugate $\bar{T}_{p_{1}, \cdots, p_{d}}$ 
is represented by a black vertex with the same above principle for its $d$ half-lines. Each index $p_{a}$ of any tensor is distinguished from the others and no symmetry is assumed between the indices. For this reason, each contraction can only occur between indices $p_{a}$ of the same sub-label or color $a$, one belonging to a given $T$ and the other to a given $\bar{T}$. This contraction manifests at the level of the graph by connecting the two half-lines of index $a$ bewteen the two vertices associated with $T$ and $\bar{T}$.

We will concentrate on rank $d=3$, as the general case $d$ recovers from this case. If one wishes to count all the possible contractions between $n$ tensors and $n$ conjugate tensors, this can be thought as counting all possible parings in the way given in Figure 3. Thus, we are enumerating

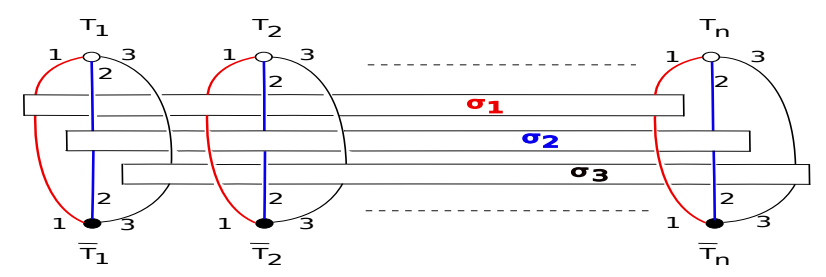

Figure 3: A tensor contraction identified as permutation triple $\left(\sigma_{1}, \sigma_{2}, \sigma_{3}\right)$.

permutation triples $\left(\sigma_{1}, \sigma_{2}, \sigma_{3}\right) \in S_{n} \times S_{n} \times S_{n}, S_{n}$ being the symmetric group of $n$ elements, up to the equivalence

$$
\left(\sigma_{1}, \sigma_{2}, \sigma_{3}\right) \sim\left(\gamma_{1} \sigma_{1} \gamma_{2}, \gamma_{1} \sigma_{2} \gamma_{2}, \gamma_{1} \sigma_{3} \gamma_{2}\right), \quad \gamma_{i} \in S_{n}
$$

This amounts to count elements of the double quotient $\operatorname{Diag}\left(S_{n}\right) \backslash\left(S_{n} \times S_{n} \times S_{n}\right) / \operatorname{Diag}\left(S_{n}\right)$. The enumeration of cosets relates to the counting of orbits of the left/right diagonal action. We therefore rely on Burnside's lemma and write, in terms of the fixed points of the same action described above,

$$
\left|H_{1} \backslash G / H_{2}\right|=\frac{1}{\left|H_{1}\right|\left|H_{2}\right|} \sum_{h_{1} \in H_{1}} \sum_{h_{2} \in H_{2}} \sum_{g \in G} \delta\left(h_{1} g h_{2} g^{-1}\right),
$$

where the $\delta$ is the usual delta function on the group, $\delta(\sigma)=1$, if $\sigma=i d$, and 0 otherwise. The number of invariants expands as

$$
\begin{aligned}
Z_{3}(n) & =\frac{1}{(n !)^{2}} \sum_{\sigma_{1,2,3} \in S_{n}} \sum_{\gamma_{1}, \gamma_{2} \in S_{n}} \delta\left(\gamma_{1} \sigma_{1} \gamma_{2}^{-1} \sigma_{1}^{-1}\right) \delta\left(\gamma_{1} \sigma_{2} \gamma_{2}^{-1} \sigma_{2}^{-1}\right) \delta\left(\gamma_{1} \sigma_{3} \gamma_{2}^{-1} \sigma_{3}^{-1}\right) \\
& =\sum_{p \vdash n} \operatorname{Sym}(p), \quad \operatorname{Sym}(p):=\prod_{i=1}^{n}\left(i^{p_{i}}\right)\left(p_{i} !\right),
\end{aligned}
$$

where the sum is performed over all partitions $p$ of $n$, denoted $p \vdash n$. Programmed in Gap, and Mathematica, one obtains the sequence [OEIS: A110143 (isomorphism of graph coverings)][73]:

$$
1 ; 4 ; 11 ; 43 ; 161 ; 901 ; 5579 ; 43206 ; 378360 ; 3742738, \ldots
$$

The appendices of [8] store the programs used for this computation. 
The above generalizes for arbitrary rank $d$ using $d$-tuples of permutations $\left(\sigma_{1}, \ldots, \sigma_{d}\right) \in$ $\left(S_{n}\right)^{\times d}$ equivalent under the diagonal action $\operatorname{Diag}\left(S_{n}\right)$ such that

$$
\left(\sigma_{1}, \ldots, \sigma_{d}\right) \sim\left(\gamma_{1} \sigma_{1} \gamma_{2}, \ldots, \gamma_{1} \sigma_{d} \gamma_{2}\right)
$$

We follow step by step the same procedure and in adapted notations and obtain the number of rank $d$ tensor invariants made with $2 n$ tensor fields as

$$
Z_{d}(n)=\sum_{p \vdash n}(\operatorname{Sym}(p))^{d-2} \quad \operatorname{Sym}(p):=\prod_{i=1}^{n}\left(i^{p_{i}}\right)\left(p_{i} !\right)
$$

Given $d$ and $n$, this number can be evaluated by a GAP or Mathematica program (see again appendices of [8]). The link between the counting tensor invariants and the counting of covers will become clearer when we will develop the permutation-TopFT formulation of the counting. Let us finally mention a word about connected invariants as the above counting includes all invariants connected and disconnected ones. To obtain connected invariants one should resort by the socalled plethystic logarithm using the Moebius-mu function. The programs allowing to reach and sequences listing the numbers of connected invariants in the same reference.

\subsection{Topological Field Theory TopFT 2}

A quick look at (3.3) shows a weighted sum of delta's functions. There is a simple physical construction, namely a topological lattice gauge theory, where permutation groups play the role of gauge groups [28] (henceforth called permutation-TopFT), that gives a sense of this expression. The topological invariance of this lattice construction illuminates the link between the counting of tensor invariants and the counting of branched covers of the 2-dimensional sphere. We start by the rank $d=3$ situation as its generalization naturally follows.

We look for a topological space leading to a permutation-TopFT whose partition function corresponds to $Z_{3}(n)$ (3.3). Consider the graph $G_{3}$ in Figure 4, which has two vertices and three edges denoted by $a, b$, and $c$. Next consider $G_{3} \times S^{1}$, which amounts to let evolve $G_{3}$ along a compactified time direction and then identifying the graph at the base of the Figure 4 with the one at the top. There are three (shaded) 2-cells of this cell-complex. To do $S_{n}$ permutation-TopFT (sometime as the lattice consists in gluing of 2-cells, we further specify $\mathrm{TopFT}_{2}$ ) on this complex, we assign $a \longrightarrow \sigma_{1}, b \longrightarrow \sigma_{2}, c \longrightarrow \sigma_{3}$ where the $\sigma_{i} \in S_{n}$. It is straighforward to check that the partition function of that TopFT is precisely $Z_{3}(n)$.

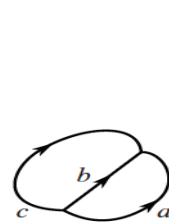

$G_{3}$

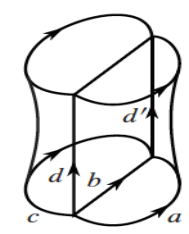

$G_{3} \times S^{1}$
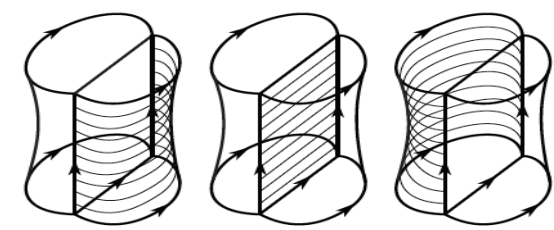

Figure 4: The underlying lattice of the $\mathrm{TopFT}_{2}$ related to $Z_{3}(n)$. 
After some manipulations (gauge fixed one $\sigma_{i}$, say $\sigma_{3}$, set $\tau_{i}=\sigma_{3}^{-1} \sigma_{i}, i=1,2$, rename $\gamma_{2} \rightarrow \gamma$, and then introduce another variable $\tau_{0}=\left(\tau_{1} \tau_{2}\right)^{-1}$ ), one arrives at:

$$
Z_{3}(n)=\frac{1}{n !} \sum_{\tau_{0}, \tau_{1}, \tau_{2} \in S_{n}} \sum_{\gamma \in S_{n}} \delta\left(\gamma \tau_{1} \gamma^{-1} \tau_{1}^{-1}\right) \delta\left(\gamma \tau_{2} \gamma^{-1} \tau_{2}^{-1}\right) \delta\left(\gamma \tau_{0} \gamma^{-1} \tau_{0}^{-1}\right) \delta\left(\tau_{0} \tau_{1} \tau_{2}\right)
$$

This formula therefore enumerates, according to the Burnside lemma, triples of permutations $\tau_{0}, \tau_{1}, \tau_{2}$ satisfying

$$
\tau_{0} \tau_{1} \tau_{2}=1
$$

More precisely, it is counting equivalence classes of these triples under the conjugation equivalence by $\gamma \in S_{n}: \tau_{i} \sim \gamma \tau_{i} \gamma^{-1}$. We recognize in (3.8) the group generated by three generators subject to one relation, which is the fundamental group of the two-sphere, with three punctures. Our counting function $Z_{3}(n)$ thus delivers the number of equivalence classes of branched covers of the 2-sphere, with 3-branch points, each equivalence class being counted once. In two dimensions, branched covers are holomorphic maps and so we expect that these permutation triples have rich properties: maps with three branch points (often taken as $0,1, \infty$ ) are called Belyi maps and are known to be definable over algebraic number fields [74]. It turns out that Belyi maps are also related to large $N$ matrix models ribbon graphs [24]. In fact, we can go deeper in this puzzling correspondence between matrices and tensors (see section 8 of [8]). Matrix and tensor models have found a clear connection: a Feynman diagram of a matrix model relates to a state/observable of a rank-3 tensor model (with a certain weight). This is a surprising feature reminiscent of dimensional reduction/uplift. This link has been deepen very recently by Amburg et al [18] as it generalizes at any $d$ (states in rank $d$ corresponds to diagrams in rank $d-1$ ). This of course deserves careful study as it may reveal important properties similar of that of gauge/gravity duality for tensor models.

Several other counting formulae for other types of tensors have been digged out in [8], for instance, the case when the tensor is fully symmetric, or a color symmetrized counting. We shall not go futher by lack of place and invite the interested reader to have a look on this work.

\subsection{Algebras and representation theoretic bases}

We will learn, in this subsection, another piece of information about the counting of complex tensor invariants under, this time, a different light, that of the representation theory of symmetric group $S_{n}$. For the basics of representation theory of the symmetric group, the reader may refer to the standard textbook by Hammermesh [75] and refer to [11] for the calculations concerning tensors (appendices therein gather all what is needed in the paper).

Mapping the counting in representations. The irreducible representations (irreps) of symmetric group $S_{n}$ are labeled by Young diagrams $R$, and these are partitions of $n: R \vdash n$. The Wigner matrices $D_{i j}^{R}(\sigma)=\langle R, j|\sigma| R, i\rangle$ defines the real matrix representation of $\sigma \in S_{n}$ in the irrep $R \vdash n$ of dimension $d(R)=n ! / h(R)$, where $h(R)$ is the product of hook-lengths in the Young diagram $R$. The matrices $D^{R}$ satisfy orthogonality properties and convolute in well-known invariants of the representation theory of $S_{n}$. We will also need the character $\chi^{R}(\cdot)=\operatorname{Tr}\left(D^{R}(\cdot)\right)$ of the representation 
$R$. Any $\delta$ on the group expands as $\delta(\cdot)=\sum_{R \vdash n}[d(R) / n !] \chi^{R}(\cdot)$. In a nutshell, the following identities hold:

$$
\begin{aligned}
& \text { orthogonality : } \quad \sum_{\sigma \in S_{n}} D_{i j}^{R}(\sigma) D_{k l}^{S}(\sigma)=\frac{n !}{d(R)} \delta^{R S} \delta_{i k} \delta_{j l} ; \\
& \text { reality : } \quad D_{i j}^{R}\left(\sigma^{-1}\right)=D_{j i}^{R}(\sigma) ; \\
& \text { Clebsch-Gordan : } \quad \sum_{\sigma \in S_{n}} D_{i_{1} j_{1}}^{R_{1}}(\sigma) D_{i_{2} j_{2}}^{R_{2}}(\sigma) D_{i_{3} j_{3}}^{R_{3}}(\sigma)=\frac{n !}{d\left(R_{3}\right)} \sum_{\tau} C_{i_{1}, i_{2} ; i_{3}}^{R_{1}, R_{2} ; R_{3}, \tau} C_{j_{1}, j_{2} ; j_{3}}^{R_{1}, R_{2}, R_{3}, \tau} \\
& \left.\tau \in\left[1, \mathrm{C}\left(R_{1}, R_{2}, R_{3}\right)\right]\right]
\end{aligned}
$$

where the symbol

$$
\mathrm{C}\left(R_{1}, R_{2}, R_{3}\right)=\frac{1}{n !} \sum_{\sigma \in S_{n}} \chi^{R_{1}}(\sigma) \chi^{R_{2}}(\sigma) \chi^{R_{3}}(\sigma)
$$

stands for the so-called Kronecker coefficient. The Kronecker coefficient is the multiplicity of the one-dimensional (trivial) representation in the tensor product $R_{1} \otimes R_{2} \otimes R_{3}$.

Expanding (3.3) in irreps, we have by a small calculation

$$
\begin{aligned}
Z_{3}(n) & =\frac{1}{(n !)^{2}} \sum_{\sigma_{i} \in S_{n}} \sum_{\gamma_{1}, \gamma_{2} \in S_{n}} \delta\left(\gamma_{1} \sigma_{1} \gamma_{2}^{-1} \sigma_{1}^{-1}\right) \delta\left(\gamma_{1} \sigma_{2} \gamma_{2}^{-1} \sigma_{2}^{-1}\right) \delta\left(\gamma_{1} \sigma_{3} \gamma_{2}^{-1} \sigma_{3}^{-1}\right) \\
& =\frac{1}{(n !)^{2}} \sum_{\gamma_{i} \in S_{n}} \sum_{R_{i} \vdash n} \chi^{R_{1}}\left(\gamma_{1}\right) \chi^{R_{1}}\left(\gamma_{2}\right) \chi^{R_{2}}\left(\gamma_{1}\right) \chi^{R_{2}}\left(\gamma_{2}\right) \chi^{R_{3}}\left(\gamma_{1}\right) \chi^{R_{3}}\left(\gamma_{2}\right) \\
& =\sum_{R_{1}, R_{2}, R_{3} \vdash n}\left(\mathrm{C}\left(R_{1}, R_{2}, R_{3}\right)\right)^{2} .
\end{aligned}
$$

The counting of rank $d=3$ observables equates to a sum of square of Kronecker coefficients (shortly called at times Kroneckers). The above identity is of a certain interest as it is a longstanding open problem to give a combinatorial sense of $\mathrm{C}\left(R_{1}, R_{2}, R_{3}\right)$ thereby ensuring its positivity (see Problem 10 in [76]; keeping in mind the way that Littlewood-Richardson coefficient have found a combinatorial description). Furthermore, this very coefficient attracks today a lot of attention in the theoretical computer science community, in particular in computational complexity theory $[77,78]$ as it turns out to be the object of interest for understanding a geometrical version of the famous problem $P$ vs $N P$. We have given above a combinatorial interpretation of a sum of squares of Kroneckers as the number of 3-regular edge-colored bipartite graphs. A question arises: is there a refinement of the counting of rank 3 observables that boils down to a single coefficient? This should be investigated. Finally, to change group invariance, namely from $U(N)$ to $O(N)$, as we will see in the next section, also impacts (3.11).

The relation (3.11) generalizes in any rank $d: Z_{d}(n)=\sum_{R_{1}, R_{2}, R_{3} \vdash n}\left(\mathrm{C}\left(R_{1}, R_{2}, \ldots, R_{d}\right)\right)^{2}$, where $\mathrm{C}\left(R_{1}, R_{2}, \ldots, R_{d}\right)$ is not the Kronecker coefficient (as this name is reserved for 3 irreps) but a generalized version of it: it counts the multiplicity of the trivial representation in the tensor product of all $R_{i}$ listed.

$\mathscr{K}_{d}(n)$, the double coset graph algebra. We now introduce an algebraic structure on observables. Such an algebra has been very fruitful in matrix models since it allows one to uncover computable sectors. Consider the group algebra $\mathbb{C}\left(S_{n}\right)$, i.e. the space of linear combinations which read $a=\sum_{\sigma \in S_{n}} \lambda_{\sigma} \sigma$, with $\lambda_{\sigma} \in \mathbb{C}$. As customary, we start by $d=3$ for simplicity. 
In the double coset formulation, we counted the orbits

$$
\left(\sigma_{1}, \sigma_{2}, \sigma_{3}\right) \sim\left(\gamma_{1} \sigma_{1} \gamma_{2}, \gamma_{1} \sigma_{2} \gamma_{3}, \gamma_{1} \sigma_{3} \gamma_{2}\right)
$$

We now want to embed these orbits in $\mathbb{C}\left(S_{n}\right)^{\otimes 3}$. For this purpose, define $\mathscr{K}_{3}(n) \subset \mathbb{C}\left(S_{n}\right)^{\otimes 3}$ as the vector space over $\mathbb{C}$ such that:

$$
\mathscr{K}_{3}(n)=\operatorname{Span}_{\mathbb{C}}\left\{\sum_{\gamma_{1}, \gamma_{2} \in S_{n}} \gamma_{1} \sigma_{1} \gamma_{2} \otimes \gamma_{1} \sigma_{2} \gamma_{2} \otimes \gamma_{1} \sigma_{3} \gamma_{2}, \sigma_{1}, \sigma_{2}, \sigma_{3} \in S_{n}\right\} .
$$

By construction, it is an obvious fact that $\operatorname{dim}_{\mathbb{C}} \mathscr{K}_{3}(n)=Z_{3}(n)$, as a generator of $\mathscr{K}_{3}(n)$ is precisely (the sum of all elements of) an orbit of the left/right diagonal group action (3.12).

The vector space $\mathscr{K}_{3}(n)$ has some properties that we now examine. Introducing a minor change by a convenient normalization, any basis element of $\mathscr{K}_{3}(n)$ expresses as

$$
A_{\sigma_{1}, \sigma_{2}, \sigma_{3}}=\frac{1}{(n !)^{2}} \sum_{\gamma_{1}, \gamma_{2} \in S_{n}} \gamma_{1} \sigma_{1} \gamma_{2} \otimes \gamma_{1} \sigma_{2} \gamma_{2} \otimes \gamma_{1} \sigma_{3} \gamma_{2}
$$

One should keep in mind that $A_{\sigma_{1}, \sigma_{2}, \sigma_{3}}$ corresponds to a graph (observable). We can multiply two such elements and obtain:

$$
A_{\sigma_{1}, \sigma_{2}, \sigma_{3}} A_{\sigma_{4}, \sigma_{5}, \sigma_{6}}=\frac{1}{n !} \sum_{\tau \in S_{n}} A_{\sigma_{1} \tau \sigma_{4}, \sigma_{2} \tau \sigma_{5}, \sigma_{3} \tau \sigma_{6}}
$$

That means that the product is stable in $\mathscr{K}_{3}(n)$, and therefore it makes it an algebra. From such a property, we infer that graphs multiply in $\mathscr{K}_{3}(n)$, which henceforth can be called a graph algebra. $\mathscr{K}_{3}(n=1) \equiv\{e\}$ is trivial. The work [11] provides the multiplication tables for $\mathscr{K}_{3}(n=2)$ (isomorphic to $\left.\mathbb{C}\left(S_{n}\right) \otimes \mathbb{C}\left(S_{n}\right)\right)$ and $\mathscr{K}_{3}(n=3)$, both are them are commutative.

It is also not difficult to realize that the product of $\mathscr{K}_{3}(n)$ is associative and admits a unit, namely the orbit containing $(i d, i d, i d)$. We conclude that $\mathscr{K}_{3}(n)$ is an associative unital subalgebra of $\mathbb{C}\left(S_{n}\right)^{\otimes 3}$. Moreover, $\mathscr{K}_{3}(n)$ is semi-simple with the nondegenerate pairing [Prop. 2, [11]]

$$
\delta_{3}\left(\otimes_{i=1}^{3} \sigma_{i} ; \otimes_{i=1}^{3} \sigma_{i}^{\prime}\right)=\prod_{i=1}^{3} \delta\left(\sigma_{i} \sigma_{i}^{\prime-1}\right) .
$$

All the above properties extend at any rank $d \geq 3: \mathscr{K}_{d}(n)$ is an associative unital semi-simple subalgebra of $\mathbb{C}\left(S_{n}\right)^{\otimes d}$. It is an interesting theme of research to determine the structure coefficients of the graph algebra $\mathscr{K}_{d}(n)$ and check if it has some known isomorphism class. The semi-simplicity property of an algebra entails, by the Wedderburn-Artin theorem, that the algebra admits a decomposition as a direct sum of matrix subalgebras. $\mathscr{K}_{d}(n)$ therefore admits a Wedderburn-Artin decomposition. In fact, from the identity $Z_{d}(n)=\sum_{R_{1}, R_{2}, R_{3} \vdash n}\left(\mathrm{C}\left(R_{1}, R_{2}, \ldots, R_{d}\right)\right)^{2}$, the dimension of each matrix subalgebra read-off as the square of the multiplicity $\left(\mathrm{C}\left(R_{1}, R_{2}, \ldots, R_{d}\right)\right)^{2}$. In the following, we identify a basis of this matrix subalgebras in rank $d=3$.

$\mathscr{K}_{3}(n)$ as a centralizer algebra. There is another formulation of the counting of rank $d$ observables by gauge fixing one $\sigma_{i}$. In rank $d=3$, we could choose to fix $\sigma_{1},\left(\sigma_{1}, \sigma_{2}, \sigma_{3}\right) \rightarrow$ $\left(1, \sigma_{1}^{-1} \sigma_{2}, \sigma_{1}^{-1} \sigma_{3}\right) \equiv\left(1, \tau_{1}, \tau_{2}\right)$. The action of $\gamma_{2}=\gamma$ reduces to $\left(\tau_{1}, \tau_{2}\right)$ as

$$
\left(\tau_{1}, \tau_{2}\right) \sim\left(\gamma \tau_{1} \gamma^{-1}, \gamma \tau_{2} \gamma^{-1}\right)
$$


In the same way as previously done, we can span the algebra $\mathscr{K}_{3}^{\prime}(n)$ over the orbits of couples $\tau_{1} \otimes \tau_{2}$. We show that it is a subalgebra of the group algebra $\mathbb{C}\left(S_{n}\right) \otimes \mathbb{C}\left(S_{n}\right)$ which is invariant under conjugation by the diagonally embedded $S_{n}$. In such a setting, we call $\mathscr{K}_{3}^{\prime}(n)$, a permutation centralizer algebra. All properties of the double coset formulation recover here as well, simply because $\mathscr{K}_{3}^{\prime}(n)$ and $\mathscr{K}_{3}(n)$ are isomorphic.

$\mathscr{K}_{3}(n)$ decomposed in matrix blocks. We investigate a base of $\mathscr{K}_{3}(d)$ which makes explicit the Wedderburn-Artin decomposition. Start by the Fourier basis of $\mathbb{C}\left(S_{n}\right)$ defined as

$$
Q_{i j}^{R}=\frac{\kappa_{R}}{n !} \sum_{\sigma \in S_{n}} D_{i j}^{R}(\sigma) \sigma
$$

where $\kappa_{R}$ is a normalization factor. Let $\rho_{L}$ and $\rho_{R}$ denote the left and right multiplications on $\mathbb{C}\left(S_{n}\right)^{\otimes 3}$, respectively. Then, we introduce the following convolution

$$
\begin{aligned}
& Q_{\tau, \tau^{\prime}}^{R_{1}, R_{2}, R_{3}}=\sum_{i_{l}, j_{l}, k} C_{i_{1}, i_{2} ; i_{3}}^{R_{1}, R_{2} ; R_{3}, \tau} C_{j_{1}, j_{2} ; j_{3}}^{R_{1}, R_{2} ; R_{3}, \tau^{\prime}} \sum_{\sigma_{1}, \sigma_{2}} \rho_{L}\left(\sigma_{1}\right) \rho_{R}\left(\sigma_{2}\right) Q_{i_{1} j_{1}}^{R_{1}} \otimes Q_{i_{2} j_{2}}^{R_{2}} \otimes Q_{i_{3} j_{3}}^{R_{3}} \\
& =\kappa_{R, S, T} \sum_{\sigma_{l} \in S_{n}} \sum_{i_{l}, j_{l}} C_{i_{1}, i_{2} ; i_{3}}^{R, S ; T, \tau_{1}} C_{j_{1}, j_{2} ; j_{3}}^{R, S T, \tau_{2}} D_{i_{1}, j_{1}}^{R}\left(\sigma_{1}\right) D_{i_{2}, j_{2}}^{S}\left(\sigma_{2}\right) D_{i_{3}, j_{3}}^{T}\left(\sigma_{3}\right) \sigma_{1} \otimes \sigma_{2} \otimes \sigma_{3} .
\end{aligned}
$$

To check that the set $\left\{Q_{\tau, \tau^{\prime}}^{R_{1}, R_{2}, R_{3}}\right\}$ forms an orthogonal matrix base of $\mathscr{K}_{3}(n)$ is the next task. Provided a rightful choice of $\kappa_{R}$, these elements multiply like matrices:

$$
Q_{\tau_{1}, \tau_{2}}^{R, S, T} Q_{\tau_{2}^{\prime}, \tau_{3}}^{R^{\prime} S^{\prime}, T^{\prime}}=\delta^{R R^{\prime}} \delta^{S S^{\prime}} \delta^{T T^{\prime}} \delta_{\tau_{2} \tau_{2}^{\prime}} Q_{\tau_{1}, \tau_{3}}^{R, S, T}
$$

Then observe that, at fixed $\left[R_{1}, R_{2}, R_{3}\right], Q_{\tau, \tau^{\prime}}^{R_{1}, R_{2}, R_{3}}$ is matrix with $\mathrm{C}\left(R_{1}, R_{2}, R_{3}\right)^{2}$ entries. The fact they are orthogonal with respect to the pairing $\delta_{3}(\cdot)(3.16)$ can be also derived in a direct manner. Thus, we can infer that this is the Wedderburn-Artin basis for $\mathscr{K}_{3}(n)$.

The scrutiny of $\mathscr{K}_{3}(n)$ reports other results concerning the existence of overcomplete bases, a nontrivial center $\mathscr{Z}\left(\mathscr{K}_{3}(n)\right)$ with base elements given by $P^{R, S, T}=\sum_{\tau} Q_{\tau, \tau}^{R, S, T} . P^{R, S, T}$ counts the number of nonvanishing Kronecker coefficients, another property which might be useful for computational complexity theory. In the next section, we engage the quantum theory and underline a few properties induced by the existence of orthogonal bases.

Correlators. At rank $d=3$, we focus the Gaussian model (2.5) and aim at calculating 1pt- and 2pt-correlators. Especially in this section, we use $N_{a}=N, a=1, \ldots, d$. Let $\mathscr{O}_{\mathbf{b}}=\mathscr{O}_{\sigma_{1}, \sigma_{2}, \sigma_{3}}$ be an observable determined by a triple $\left(\sigma_{1}, \sigma_{2}, \sigma_{3}\right)$. By the Wick theorem, we obtain

$$
\left\langle\mathscr{O}_{\sigma_{1}, \sigma_{2}, \sigma_{3}}\right\rangle=\sum_{\mu \in S_{n}} N^{\mathbf{c}\left(\mu \sigma_{1}\right)+\mathbf{c}\left(\mu \sigma_{2}\right)+\mathbf{c}\left(\mu \sigma_{3}\right)}
$$

$\mathbf{c}(\alpha)$ is the number of cycles of $\alpha$. We map this expression in the Fourier components by contracting it with characters, at fixed $\left(S_{1}, S_{2}, S_{3}\right)$ :

$$
\left\langle\mathscr{O}_{S_{1}, S_{2}, S_{3}}\right\rangle=\frac{1}{(n !)^{3}} \sum_{\sigma_{l} \in S_{n}} \chi^{S_{1}}\left(\sigma_{1}\right) \chi^{S_{2}}\left(\sigma_{2}\right) \chi^{S_{3}}\left(\sigma_{3}\right)\left\langle\mathscr{O}_{\sigma_{1}, \sigma_{2}, \sigma_{3}}\right\rangle
$$




$$
=\frac{1}{(n !)^{2}}\left[\prod_{l=1}^{3} f_{N}\left(S_{l}\right)\right] \mathrm{C}\left(S_{1}, S_{2}, S_{3}\right)
$$

where $f_{N}(R)$ is the product of box weights after filling $R$. Hence, the correlators $\left\langle\mathscr{O}_{S_{1}, S_{2}, S_{3}}\right\rangle$ are proportional to the Kronecker coefficients. A similar expression hold for rank $d 1 \mathrm{pt}$-function, where the Kronecker is replaced by the coefficient $\mathrm{C}\left(S_{1}, S_{2}, \ldots, S_{d}\right)$. Hence using software programs (in particular Sage has a useful and efficient package to compute Kroneckers), we can compute explicitly this sector of the Gaussian tensor model, and that is quite remarkable.

The last thing that we wish to sketch is that normal ordered 2 pt-functions evaluate wtih the Wedderburn-Artin base $\left\{Q_{\tau, \tau^{\prime}}^{R, S, T}\right\}$. We have (see equation (97) in [11]):

$$
\left\langle\mathscr{O}_{\sigma_{1}, \sigma_{2}, \sigma_{3}} \mathscr{O}_{\tau_{1}, \tau_{2}, \tau_{3}}\right\rangle=\sum_{\gamma_{1}, \gamma_{2}} N^{3 n} \delta_{3}\left[\left(\sigma_{1} \otimes \sigma_{2} \otimes \sigma_{3}\right) \gamma_{1}^{\otimes 3}\left(\tau_{1} \otimes \tau_{2} \otimes \tau_{3}\right) \gamma_{2}^{\otimes 3}\left(\Omega_{1} \otimes \Omega_{2} \otimes \Omega_{3}\right)\right]
$$

where $\Omega_{i}=\sum_{\alpha_{i} \in S_{n}} N^{\mathbf{c}\left(\alpha_{i}\right)-n} \alpha_{i}$ are shown to be central elements of $\mathbb{C}\left(S_{n}\right)$. Now, we introduce the Fourier (or representation basis ) of the observables:

$$
\mathscr{O}_{\tau_{1}, \tau_{2}}^{R, S, T}=\sum_{\sigma_{1}, \sigma_{2}, \sigma_{3}} \delta_{3}\left(Q_{\tau_{1}, \tau_{2}}^{R, S, T} \sigma_{1}^{-1} \otimes \sigma_{2}^{-1} \otimes \sigma_{3}^{-1}\right) \mathscr{O}_{\sigma_{1}, \sigma_{2}, \sigma_{3}}
$$

A calculation leads to

$$
\left\langle\mathscr{O}_{\tau_{1}, \tau_{1}^{\prime}}^{R_{1}, S_{1}, T_{1}} \overline{\mathscr{O}_{\tau_{2}, \tau_{2}^{\prime}}^{R_{2}, S_{2}, T_{2}}}\right\rangle=c\left(R_{1}, S_{1}, T_{1}\right) \delta_{R_{1}, R_{2}} \delta_{S_{1}, S_{2}} \delta_{T_{1}, T_{2}} \delta_{\tau_{1}, \tau_{2}} \delta_{\tau_{1}^{\prime}, \tau_{2}^{\prime}},
$$

with $c\left(R_{1}, S_{1}, T_{1}\right)$ a constant depending on representation indices. This demonstrates that $\left\{\mathscr{O}_{\tau, \tau^{\prime}}^{R, S, T}\right\}$ forms an orthogonal basis for Gaussian normal ordered correlators arising directly from the $Q_{\tau, \tau^{\prime}}^{R, S, T}$, which are the representation theoretic base elements of $\mathscr{K}_{3}(n)$. We emphasize that more results and the proofs of above identities are available in [11]. Concerning correlators, we obtain new correspondences with cover countings in a TopFT 2 . On another side, a thorough exploration of the color symmetrized counting exhibit a graded algebra structure with grade labeled by the irreps of $S_{d}$, the group of permutation of the colors $[[1, d]$.

\section{Counting real tensor model observables}

This section deals with $O(N)$ invariants for real tensor models. We adopt the same methodology of the previous section. This section reports some of the main results of [7].

\subsection{Counting}

Tensors in this part are real and still denoted $T_{p_{1}, \ldots, p_{d}}$. Seeking a graphical representation for the contractions of tensors, we use the same scheme as in subsection 3.1.

Orthogonal invariants bijectively correspond to $d$-regular colored graphs (no bipartiteness) [43]. Indeed, at the contrary of the previous section, there is no objection that $T$ contracts its indices with another $T$. However, the bipartiteness is still useful to achieve a counting. A way to restore this property consists in the insertion of another type of vertex of valence 2, called black vertex, on each edge of the graph. We keep the initial vertices associated with $T$ as white. Consider the contraction 
of $2 n$ tensors or white vertices and complete the graph associated with the contraction. Then add on each edge the black vertices denoted $v_{i}^{j}, i=1, \cdots, 2 n$ and $j=1, \cdots, d$. The resulting graph is neither regular, nor properly edge-colored. It is however bipartite. We illustrate the contraction of $2 n$ tensors in rank 3 by the diagram of Figure 5 .

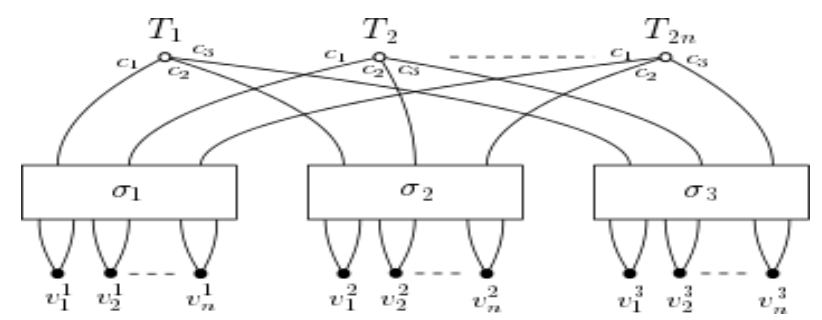

Figure 5: Diagrammatics for real tensor contractions.

The number of contractions matches with the number of permutation triples $\left(\sigma_{1}, \sigma_{2}, \sigma_{3}\right) \in$ $S_{2 n} \times S_{2 n} \times S_{2 n}$ subjected to the equivalence

$$
\left(\sigma_{1}, \sigma_{2}, \sigma_{3}\right) \sim\left(\gamma_{1} \sigma_{1} \gamma, \gamma_{2} \sigma_{2} \gamma, \gamma_{3} \sigma_{3} \gamma\right), \quad \gamma_{i} \in S_{n}\left[S_{2}\right], \gamma \in S_{n}
$$

where $S_{n}\left[S_{2}\right]$ is the so-called wreath product of $S_{n}$ by $S_{2}$ (that is the semi direct product $S_{n} \ltimes\left(S_{2}\right)^{n}$ ). Its action describes as follows: $\left(S_{2}\right)^{n}$ permutes independently the half-lines of the $n v_{i}^{j}$, s; then, $S_{n}$ permutes the $n$ vertices $v_{i}^{j}$ of a given color $j$, hence the three copies of the wreath products in rank 3.

Up to equivalence, any contraction belongs to the double quotient

$$
S_{n}\left[S_{2}\right] \times S_{n}\left[S_{2}\right] \times S_{n}\left[S_{2}\right] \backslash\left(S_{2 n} \times S_{2 n} \times S_{2 n}\right) / \operatorname{Diag}\left(S_{2 n}\right) .
$$

The computation of the cardinality of double coset requires a technique different from the complex case but it still involves the Burnside lemma. Its last stage makes use of software programs. The number of invariants in rank $d=3$, that we denote $Z_{3}^{o}(2 n)$, follows the sequence:

$$
1 ; 5 ; 16 ; 86 ; 448 ; 3580 ; 34981 ; 448628 ; 6854130 ; 121173330 \text {. }
$$

Note that Read [79] introduced orthogonal polynomial techniques to achieve the same counting. He was able to obtain the first three terms of the sequence in his seminal paper.

The generalization at any rank $d$ is straightforward. Connected tensor invariants can be also generated by the plethystic logarithm. At rank 3, one gets

$$
1 ; 4 ; 11 ; 60 ; 318 ; 2806 ; 29359 ; 396196 ; 6231794 ; 112137138 \text {. }
$$

Other counting sequences at rank $d=4$ are provided in [7], and its appendices list Mathematica programs computing both general and connected sequences at arbitrary $d$. To our knowledge, none of the sequences at $d \geq 4$ are yet reported in OEIS. 


\subsection{Topological Field Theory TopFT 2}

TopFT interprets the tensor invariant counting in a different manner. Consider the counting of classes in the double coset (4.2), and the relation (4.1). Using Burnside's lemma, one infers that

$$
Z_{3}^{o}(2 n)=\frac{1}{\left[n !(2 !)^{n}\right]^{3}(2 n) !} \sum_{\gamma_{i} \in S_{n}\left[S_{2}\right]} \sum_{\sigma_{i} \in S_{2 n}} \sum_{\gamma \in S_{2 n}} \delta\left(\gamma_{1} \sigma_{1} \gamma \sigma_{1}^{-1}\right) \delta\left(\gamma_{2} \sigma_{2} \gamma \sigma_{2}^{-1}\right) \delta\left(\gamma_{3} \sigma_{3} \gamma \sigma_{3}^{-1}\right),
$$

with $\delta$ the Kronecker symbol on $S_{2 n}$.

We identify the above counting as a partition function of a $\mathrm{TopFT}_{2}$ on a cellular complex given by Figure 6. Two gauge groups $S_{2 n}$ and $S_{n}\left[S_{2}\right]$ are needed on this lattice. Associated with that, three cylinders sharing one of their boundary circle characterize the topology of that 2-complex. The enumeration of 3-index orthogonal invariants corresponds to a $S_{2 n}$-TopFT 2 on 3 glued cylinders along one circle, with a restriction such that, the opposite boundary circle is associated with a generator of the gauge group $S_{n}\left[S_{2}\right]$. Such a topological theory has boundary holonomies decorated with $S_{n}\left[S_{2}\right]$ group elements.
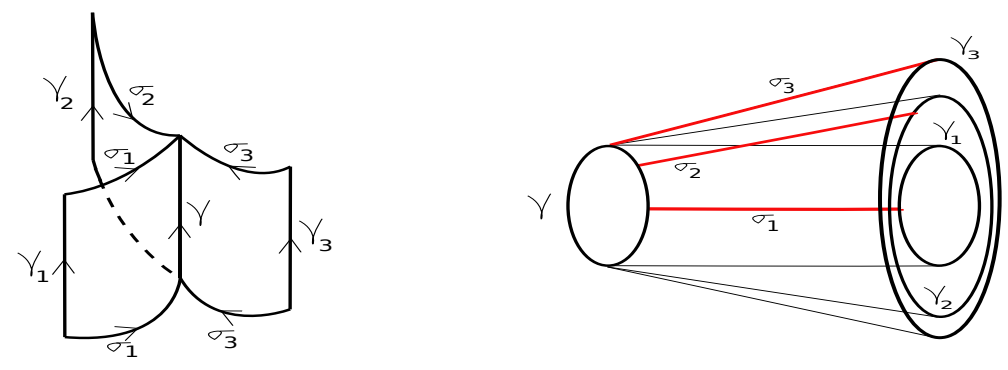

Figure 6: Underlying lattice of TopFT2 related to $Z_{3}^{o}(2 n)$.

After a few derivations involving integration and changes of variables, we come to

$$
Z_{3}^{o}(2 n)=\frac{1}{\left[n !(2 !)^{n}\right]^{3}} \sum_{\gamma_{i} \in S_{n}\left[S_{2}\right]} \sum_{\sigma_{1,2} \in S_{2 n}} \delta\left(\gamma_{1} \sigma_{1} \gamma_{3} \sigma_{1}^{-1}\right) \delta\left(\gamma_{2} \sigma_{2} \gamma_{3} \sigma_{2}^{-1}\right)
$$

This integration pictures as in Figure 7 as the removal of a 1-cell associated with the variable $\gamma$ in the 2-complex. The partition function therefore shows two types of invariances: the extraction of $\gamma$ corresponds to one type of topological invariance, and then, it is followed by the change of variables $\sigma_{1,2} \rightarrow \sigma_{1,2} \sigma_{3}^{-1}$ corresponding to a topological invariance of a second kind. Thus, the partition function (4.6) finds another form as

$$
Z_{3}(2 n)=Z\left(S^{1} \times I ;\left(D_{S_{n}\left[S_{2}\right]}\right)^{\times 3}\right),
$$

where the right-hand-side defines the partition function obtained by inserting $3 S_{n}\left[S_{2}\right]$-defects, one at each end of the cylinder $S^{1} \times I$, and another one at finite time $t_{0} \in I$, see Figure 8 . A defect is defined as a closed non-intersecting loop with a marked point. The relation (4.7) shows that orthogonal invariants are in one-to-one correspondence with $n$-fold covers of the cylinder with 3 defects, up to a (symmetry) factor, the stabilizer subgroup of the graph that we denote $\operatorname{Aut}\left(G_{\sigma_{1}, \sigma_{2}, \sigma_{3}}\right)$. The order of the stabilizer infers from $\operatorname{Aut}\left(G_{\sigma_{1}, \sigma_{2}, \sigma_{3}}\right)=\sum_{\gamma_{i} \in S_{n}\left[S_{2}\right]} \delta\left(\gamma_{1} \sigma_{1} \gamma_{3} \sigma_{1}^{-1}\right) \delta\left(\gamma_{2} \sigma_{2} \gamma_{3} \sigma_{2}^{-1}\right)$. This 

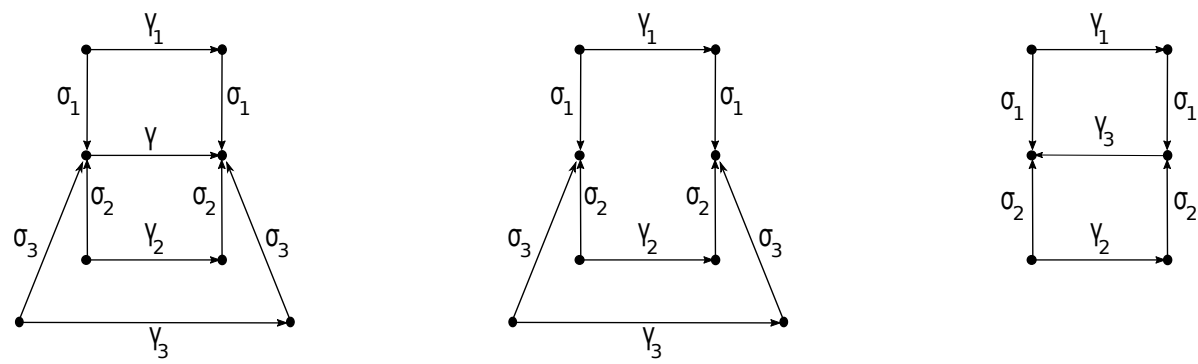

Figure 7: Topological transformations leaving the partition function invariant.

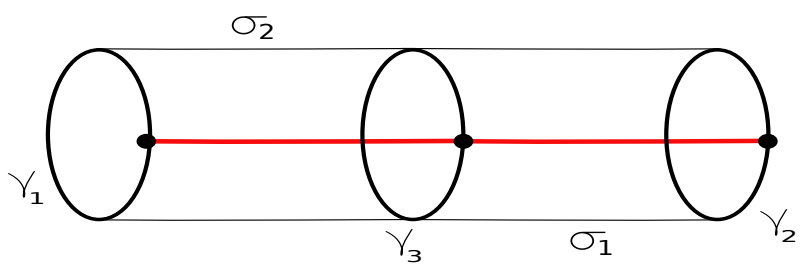

Figure 8: Cylinder with 3 defects.

number meets the number of equivalences $\left(S_{n}\left[S_{2}\right] \times S_{n}\left[S_{2}\right]\right) \backslash\left(S_{n} \times S_{n}\right) / \operatorname{Diag}\left(S_{n}\left[S_{2}\right]\right)$ corresponding to a fixed $\left(\sigma_{1}, \sigma_{2}\right)$.

The rank $d$ case tracks the same logic: the counting expresses in terms of $n$-covers of $d-1$ cylinders with $d$ defects, one defect shared by all cylinders. It is noteworthy that TopFT 2 may enrich the counting orthogonal invariants with a geometrical picture. Indeed, the base space of the TopFT is generally regarded as a string worldsheet [80,81]. The counting becomes now counting of worldsheet maps over a cylinder with defects. Once more, this intimates that a link may exist between tensor models and string theory, which TopFT could elucidate. This merits full-fledged treatment.

\subsection{Algebras and representation theoretic bases}

We address the algebraic structure underlying the counting of real tensor invariants. First, we switch to representation theory that delivers a different expression of the same enumeration. Then, this new reading allows us to motivate the search for an algebra making sense of the new formula. Careful attention must be paid on the fact that we might use the same notation as in subsection 3.3 while we are dealing with $S_{2 n}$ (and not $S_{n}$ ).

Mapping the counting in representations. Let us revisit the counting (4.5) using the representation theory of the symmetric group $S_{2 n}$ (the appendices of [7] reviews the main identities used in the following). The partitions of $2 n$ or Young diagrams, $R \vdash 2 n$, label irreps of $S_{2 n}$.

The counting (4.5) expands as

$$
\begin{aligned}
Z_{3}^{o}(2 n) & =\frac{1}{\left[n !(2 !)^{n}\right]^{3}(2 n) !} \sum_{\gamma_{l} \in S_{n}\left[S_{2}\right]} \sum_{\gamma \in S_{2 n} R_{l} \vdash 2 n} \chi^{R_{1}}\left(\gamma_{1}\right) \chi^{R_{1}}(\gamma) \chi^{R_{2}}\left(\gamma_{2}\right) \chi^{R_{2}}(\gamma) \chi^{R_{3}}\left(\gamma_{3}\right) \chi^{R_{3}}(\gamma) \\
& =\frac{1}{\left[n !(2 !)^{n}\right]^{3}} \sum_{R_{l} \vdash 2 n} \mathbf{C}\left(R_{1}, R_{2}, R_{3}\right)\left[\sum_{\gamma_{1} \in S_{n}\left[S_{2}\right]} \chi^{R_{1}}\left(\gamma_{1}\right)\right]\left[\sum_{\gamma_{2} \in S_{n}\left[S_{2}\right]} \chi^{R_{2}}\left(\gamma_{2}\right)\right],
\end{aligned}
$$


where the Kronecker coefficient of $S_{2 n}$ is defined by

$$
\mathbf{C}\left(R_{1}, R_{2}, R_{3}\right)=\frac{1}{(2 n) !} \sum_{\gamma \in S_{2 n}} \chi^{R_{1}}(\gamma) \chi^{R_{2}}(\gamma) \chi^{R_{3}}(\gamma)
$$

The two other factors $\sum_{\gamma_{i} \in S_{n}\left[S_{2}\right]} \chi^{R_{i}}\left(\gamma_{i}\right), i=1,2$, need a non-obvious treatment. To tackle these sums, we use a result by Howe [82] (see also [83, 84, 85, 29]): $\sum_{\gamma \in S_{n}\left[S_{2}\right]} \chi^{R}(\gamma)=\left|S_{n}\left[S_{2}\right]\right| m^{R}$, where $m^{R}=1$ if $R$ is an "even" partition, and $m^{R}=0$ otherwise. A partition is called even if all its row lengths are even. Inserting this in (4.8), we have

$$
Z_{3}^{o}(2 n)=\sum_{R_{l} \vdash 2 n / R_{l} \text { is even }} \mathbf{C}\left(R_{1}, R_{2}, R_{3}\right) .
$$

A Sage code implements the sum (4.10) produces the sequence (4.3) as expected.

$Z_{3}^{o}(2 n)$ is also the dimension of an algebra $\mathscr{K}_{3}(n)$. We will make this clear in the next developments. Here again, we realize that counting of colored graphs could contribute to the famous problem of giving a combinatorial interpretation to the Kronecker coefficients [77, 78]. From the previous section, it was shown that the sum of squares of Kronecker coefficients associated with $S_{n}$ equals the number of $d$-regular bipartite colored graphs made with $n$ black and $n$ white vertices. Here, the interpretation goes as follows: the number of $d$-regular colored graphs (not necessarily bipartite) equals the sum of all "even" Kroneckers of $S_{2 n}$. The two countings are obviously radically different. On one hand, the real case associated with $S_{2 n}$ have more terms but a linear power of the Kroneckers. On the other hand, we may associate the bipartite structure (or complex tensors) to the presence of squares in the sum of Kroneckers. Switching from $U(N)$ to $O(N)$, in other words from complex to real, we get rid of the squares but need to deal with a sum over a much larger number of terms. So is there a real Lie group having having even a lesser number of terms than $O(N)$ ?

We may finally add that the above counting extends to arbitrary $d$ :

$$
Z_{d}^{o}(2 n)=\sum_{R_{l} \vdash 2 n / R_{l} \text { is even }} \mathbf{C}_{d}\left(R_{1}, \ldots, R_{d}\right),
$$

where $\mathbf{C}_{d}\left(R_{1}, \ldots, R_{d}\right)$ should have now an obvious meaning. This expression can be computationally implemented without any issue with Sage.

$\mathscr{K}_{d}^{o}(n)$, the double coset graph algebra. Let us focus on the algebra generated by real tensor invariants. We will consider $\mathbb{C}\left(S_{2 n}\right)$, the group algebra of $S_{2 n}$. We first study the rank 3 before achieving the general case.

We fix $d=3$. Consider $\sigma_{1} \otimes \sigma_{2} \otimes \sigma_{3}$ as an element of the group algebra $\mathbb{C}\left(S_{2 n}\right)^{\otimes 3}$, and three left actions of the subgroup $S_{n}\left[S_{2}\right]$ and the diagonal right action $\operatorname{Diag}\left(\mathbb{C}\left(S_{2 n}\right)\right)$ on this triple as:

$$
\sigma_{1} \otimes \sigma_{2} \otimes \sigma_{3} \rightarrow \sum_{\gamma_{i} \in S_{n}\left[S_{2}\right]} \sum_{\gamma \in S_{2 n}} \gamma_{1} \sigma_{1} \gamma \otimes \gamma_{2} \sigma_{2} \gamma \otimes \gamma_{3} \sigma_{3} \gamma
$$

$\mathscr{K}_{3}^{o}(2 n)$ is the vector subspace of $\mathbb{C}\left(S_{2 n}\right)^{\otimes 3}$ which is invariant under these subgroup actions:

$$
\mathscr{K}_{3}^{o}(2 n)=\operatorname{Span}_{\mathbb{C}}\left\{\sum_{\gamma_{i} \in S_{n}\left[S_{2}\right]} \sum_{\gamma \in S_{2 n}} \gamma_{1} \sigma_{1} \gamma \otimes \gamma_{2} \sigma_{2} \gamma \otimes \gamma_{3} \sigma_{3} \gamma, \quad \sigma_{1}, \sigma_{2}, \sigma_{3} \in S_{2 n}\right\} .
$$


It is obvious that $\operatorname{dim} \mathscr{K}_{3}^{o}(2 n)=Z_{3}^{o}(2 n)$, since each base element represents a graph equivalence class counted once in $Z_{3}^{o}(2 n)$. Pick two base elements and multiply them

$$
\begin{aligned}
& {\left[\sum_{\gamma_{i} \in S_{n}\left[S_{2}\right]} \sum_{\gamma \in S_{2 n}} \gamma_{1} \sigma_{1} \gamma \otimes \gamma_{2} \sigma_{2} \gamma \otimes \gamma_{3} \sigma_{3} \gamma\right]\left[\sum_{\tau_{i} \in S_{n}\left[S_{2}\right]} \sum_{\tau \in S_{2 n}} \tau_{1} \sigma_{1}^{\prime} \tau \otimes \tau_{2} \sigma_{2}^{\prime} \tau \otimes \tau_{3} \sigma_{3}^{\prime} \tau\right]} \\
& =\sum_{\tau_{i} \in S_{n}\left[S_{2}\right]} \sum_{\gamma \in S_{2 n}}\left[\sum_{\gamma_{i} \in S_{n}\left[S_{2}\right]} \sum_{\tau \in S_{2 n}} \gamma_{1}\left(\sigma_{1} \gamma \tau_{1} \sigma_{1}^{\prime}\right) \tau \otimes \gamma_{2}\left(\sigma_{2} \gamma \tau_{2} \sigma_{2}^{\prime}\right) \tau \otimes \gamma_{3}\left(\sigma_{3} \gamma \tau_{3} \sigma_{3}^{\prime}\right) \tau\right] .
\end{aligned}
$$

This shows that the multiplication is stable in $\mathscr{K}_{3}^{o}(2 n)$, and hence, $\mathscr{K}_{3}^{o}(2 n)$ forms graph algebra. In the same vein as before, we can also show that $\mathscr{K}_{3}^{o}(2 n)$ is an associative, unital subalgebra of $\mathbb{C}\left(S_{2 n}\right)^{\otimes 3}$ which is semi-simple under a similar nondegenerate pairing $\delta_{3}(\cdot, \cdot)$, product of delta's on each factor of $\mathbb{C}\left(S_{2 n}\right)^{\otimes 3}$, see (3.16). The proof is totally similar for $\mathscr{K}_{d}^{o}(2 n)$ (considering $d$ factors in the tensor product) that is also a semi-simple associative unital subalgebra of $\mathbb{C}\left(S_{2 n}\right)^{\otimes d}$ of dimension $Z_{d}^{o}(2 n)$.

The semi-simplicity ensures that, by the Wedderburn-Artin theorem, $\mathscr{K}_{d}^{o}(2 n)$ decomposes in matrix subalgebras. In the complex case and at rank $d=3$, we successfully exhibit the orthogonal base making apparent this matrix decomposition. The real case appears more difficult to handle and, so far, no Wedderburn-Artin base has been found for $d \geq 3$. Postponing this for future investigations, it remains an issue that we could certainly address: in rank $d=3$, find a representation base with labels that reflect the dimension (4.10). This is our next goal.

A representation theoretic base for $\mathscr{K}_{3}^{o}(2 n)$. We start by considering (3.18) for the present case, and so replace $S_{n}$ by $S_{2 n}, \kappa_{R}$ is a different but fixed constant, such that $\left\{Q_{i j}^{R}\right\}$ forms this time an orthonormal base of $\mathbb{C}\left(S_{2 n}\right): \delta\left(Q_{i j}^{R} ; Q_{i^{\prime} j^{\prime}}^{R^{\prime}}\right)=\delta_{R R^{\prime}} \delta_{i i^{\prime}} \delta_{j j^{\prime}}$.

Consider the right diagonal action $\rho_{R}(\cdot)$ and the three left actions $\rho_{i}(\cdot)$ on the tensor product $\mathbb{C}\left[S_{2 n}\right]^{\otimes 3}$. We write:

$$
\begin{aligned}
& \sum_{\gamma_{1}, \gamma_{2}, \gamma_{3} \in S_{n}\left[S_{2}\right]} \sum_{\gamma \in S_{2 n}} \rho_{1}\left(\gamma_{1}\right) \rho_{2}\left(\gamma_{2}\right) \rho_{3}\left(\gamma_{3}\right) \rho_{R}(\gamma) Q_{i_{1} j_{1}}^{R_{1}} \otimes Q_{i_{2} j_{2}}^{R_{2}} \otimes Q_{i_{3} j_{3}}^{R_{3}} \\
& =\frac{(2 n) !}{d\left(R_{3}\right)} \sum_{\gamma_{a}} \sum_{p_{l}, q_{l}} \sum_{\tau} C_{j_{1}, j_{2} ; j_{3}}^{R_{1}, R_{2} ; R_{3}, \tau} C_{q_{1}, q_{2} ; q_{3}}^{R_{1}, R_{2} ; R_{3}, \tau} D_{p_{1} i_{1}}^{R_{1}}\left(\gamma_{1}\right) D_{p_{2} i_{2}}^{R_{2}}\left(\gamma_{2}\right) D_{p_{3} i_{3}}^{R_{3}}\left(\gamma_{3}\right) Q_{p_{1} q_{1}}^{R_{1}} \otimes Q_{p_{2} q_{2}}^{R_{2}} \otimes Q_{p_{3} q_{3}}^{R_{3}} .
\end{aligned}
$$

Overlapping the result with a Glebsch-Gordan coefficent yields

$$
\begin{aligned}
& \sum_{j_{l}} C_{j_{1}, j_{2} ; j_{3}}^{R_{1}, R_{2} ; R_{3}, \tau} \sum_{\gamma_{a}} \sum_{\gamma} \rho_{1}\left(\gamma_{1}\right) \rho_{2}\left(\gamma_{2}\right) \rho_{3}\left(\gamma_{3}\right) \rho_{R}(\gamma) Q_{i_{1} j_{1}}^{R_{1}} \otimes Q_{i_{2} j_{2}}^{R_{2}} \otimes Q_{i_{3} j_{3}}^{R_{3}} \\
& =(2 n) ! \sum_{p_{l}, q_{l}} C_{q_{1}, q_{2} ; q_{3}}^{R_{1}, R_{3} ; R_{3}, \tau} \sum_{\gamma_{1}} D_{p_{1} i_{1}}^{R_{1}}\left(\gamma_{1}\right) \sum_{\gamma_{2}} D_{p_{2} i_{2}}^{R_{2}}\left(\gamma_{2}\right) \sum_{\gamma_{3}} D_{p_{3} i_{3}}^{R_{3}}\left(\gamma_{3}\right) Q_{p_{1} q_{1}}^{R_{1}} \otimes Q_{p_{2} q_{2}}^{R_{2}} \otimes Q_{p_{3} q_{3}}^{R_{3}} .
\end{aligned}
$$

An emphasis should be put on the fact that $\sum_{\gamma \in S_{n}\left[S_{2}\right]} D_{p q}^{R}(\gamma) \neq 0$, if and only if $R$ is a partition of $2 n$ with even rows. Next, the Wigner matrix element must split using the so-called branching coefficients of $S_{n}\left[S_{2}\right]$ in $S_{2 n}$. To proceed with that consider $V^{R}$ an irreps $S_{2 n}$, and the subgroup inclusion $S_{n}\left[S_{2}\right] \subset S_{2 n}$, we can decompose $V^{R}$ in irreps $V^{r}$ of $S_{n}\left[S_{2}\right]$ as $V^{R}=\oplus_{r} V^{r} \otimes V_{R, r}$, where $V_{R, r}$ is a vector space of dimension the multiplicity of the irreps $r$ in $R$. A state in this decomposition is written $\left|r, m_{r}, v_{r}\right\rangle$, where $m_{r}$ labels the states of $V^{r}$ and $v_{r}=1, \ldots, \operatorname{dim} V_{R, r}$. The branching coefficients are defined by the overlap of $\left|r, m_{r}, v_{r}\right\rangle$ with an orthonormal base of $R$ :

$$
B_{i ; m_{r}}^{R ; r, v_{r}}=\left\langle R, i \mid r, m_{r}, v_{r}\right\rangle=\left\langle r, m_{r}, v_{r} \mid R, i\right\rangle .
$$


The properties of $B_{i ; m_{r}}^{R ; r, v_{r}}$ have been listed in [7] (see page 17,18). First, decompose the above (4.16), exploiting the branching coefficient

$$
\begin{aligned}
& \sum_{j_{l}} C_{j_{1}, j_{2} ; j_{3}}^{R_{1}, R_{2} ; R_{3}, \tau} \sum_{\gamma_{a}} \sum_{\gamma} \rho_{1}\left(\gamma_{1}\right) \rho_{2}\left(\gamma_{2}\right) \rho_{3}\left(\gamma_{3}\right) \rho_{R}(\gamma) Q_{i_{1} j_{1}}^{R_{1}} \otimes Q_{i_{2} j_{2}}^{R_{2}} \otimes Q_{i_{3} j_{3}}^{R_{3}} \\
& =(2 n) !\left(n ! 2^{n}\right)^{3} B_{i_{1}}^{R_{1} ; t r} B_{i_{2}}^{R_{2} ; t r} B_{i_{3}}^{R_{3} ; t r} \sum_{p_{l}, q_{l}} C_{q_{1}, q_{2} ; q_{3}}^{R_{1}, R_{2} ; R_{3}, \tau} B_{p_{1}}^{R_{1} ; t r} B_{p_{2}}^{R_{2} ; t r} B_{p_{3}}^{R_{3} ; t r} Q_{p_{1} q_{1}}^{R_{1}} \otimes Q_{p_{2} q_{2}}^{R_{2}} \otimes Q_{p_{3} q_{3}}^{R_{3}},
\end{aligned}
$$

and then we are in position to define the representation base that we are looking for:

$$
\begin{aligned}
& Q^{R_{1}, R_{2}, R_{3}, \tau}=\kappa_{\vec{R}} \sum_{p_{l}, q_{l}} C_{q_{1}, q_{2} ; q_{3}}^{R_{1}, R_{2} ; R_{3}, \tau} B_{p_{1}}^{R_{1} ; t r} B_{p_{2}}^{R_{2} ; t r} B_{p_{3}}^{R_{3} ; t r} Q_{p_{1} q_{1}}^{R_{1}} \otimes Q_{p_{2} q_{2}}^{R_{2}} \otimes Q_{p_{3} q_{3}}^{R_{3}} \\
& =\kappa_{\vec{R}} \frac{\kappa_{R_{1}} \kappa_{R_{2}} \kappa_{R_{3}}}{((2 n) !)^{3}} \sum_{\sigma_{i}} \sum_{p_{l}, q_{l}} C_{q_{1}, q_{2} ; q_{3}}^{R_{1}, R_{2} ; R_{3}, \tau}\left[\prod_{i=1}^{3} B_{p_{i}}^{R_{i} ; t r} D_{p_{i} q_{i}}^{R_{i}}\left(\sigma_{i}\right)\right] \sigma_{1} \otimes \sigma_{2} \otimes \sigma_{3},
\end{aligned}
$$

where $\kappa_{\vec{R}}$ is a normalization constant to be fixed later on, and $\vec{R}=\left(R_{1}, R_{2}, R_{3}\right)$. Note that the set $\left\{Q^{R_{1}, R_{2}, R_{3}, \tau}\right\}$ is of cardinality the counting of orthogonal invariants (4.10). Some calculations show that the elements of $\left\{Q^{R_{1}, R_{2}, R_{3}, \tau}\right\}$ obey the invariance

$$
\left(\gamma_{1} \otimes \gamma_{2} \otimes \gamma_{3}\right) Q^{R_{1}, R_{2}, R_{3}, \tau}(\gamma \otimes \gamma \otimes \gamma)=Q^{R_{1}, R_{2}, R_{3}, \tau},
$$

and are orthonomal under the pairing

$$
\delta\left(Q^{R_{1}, R_{2}, R_{3}, \tau} ; Q^{R_{1}^{\prime}, R_{2}^{\prime}, R_{3}^{\prime}, \tau^{\prime}}\right)=\kappa_{\vec{R}}^{2} d\left(R_{3}\right) \sum_{p_{l}}\left[\prod_{i=1}^{3} B_{p_{i}}^{R_{i} ; t r}\right]^{2} \delta_{\vec{R} \vec{R}^{\prime}} \delta_{\tau \tau^{\prime}}=\kappa_{\vec{R}}^{2} d\left(R_{3}\right) \delta_{\vec{R} \vec{R}^{\prime}} \delta_{\tau \tau^{\prime}}
$$

They form in fact an orthonormal base of $\mathscr{K}_{3}^{o}(2 n)$ for a well chosen $\kappa_{\vec{R}}$. Nevertheless, they do not multiply like matrices. Indeed,

$$
Q^{R_{1}, R_{2}, R_{3}, \tau} Q^{R_{1}^{\prime}, R_{2}^{\prime}, R_{3}^{\prime}, \tau^{\prime}}=\delta_{\vec{R} \vec{R}^{\prime}} k\left(\vec{R}^{\prime}, \tau\right) Q^{R_{1}^{\prime}, R_{2}^{\prime}, R_{3}^{\prime}, \tau^{\prime}},
$$

with a factor $k\left(\vec{R}^{\prime}, \tau\right)$ that prevents their orthogonality with respect to the multiplication. They do not define the base of Wedderburn-Artin matrix decomposition. The base $\left\{Q^{R_{1}, R_{2}, R_{3}, \tau}\right\}$ decomposes $\mathscr{K}_{3}^{o}(2 n)$ in blocks mutually orthogonal in the labels $\left(R_{1}, R_{2}, R_{3}\right)$ but not in all the remaining labels.

Correlators. The calculation of correlators happens to be more involved than the bipartite complexe case. In general, correlators build a polynomial in a variable $N$, i.e. the range of a single tensor index, with powers the number cycles of some permutations. To understand and list these permutations is the first difficult point to overcome.

We shall focus on $d=3$ and Wick's theorem allows us to write the 1pt-function of an observable $O_{\mathbf{b}}=O_{\sigma_{1}, \sigma_{2}, \sigma_{3}}$ as

$$
\left\langle O_{\sigma_{1}, \sigma_{2}, \sigma_{3}}\right\rangle=\sum_{\mu \in S_{2 n}^{*}} N^{\sum_{i=1}^{3} \mathbf{c}\left(\mu \widetilde{\sigma}_{i}\right)},
$$

where $S_{2 n}^{*}$ is the subset defined by the pairings of $S_{2 n}$, that are permutations made only of transpositions and

$$
\left(\widetilde{\sigma}_{1}, \widetilde{\sigma}_{2}, \widetilde{\sigma}_{3}\right)=\left(\sigma_{1}^{-1} \xi \sigma_{1}, \sigma_{2}^{-1} \xi \sigma_{2}, \sigma_{3}^{-1} \xi \sigma_{3}\right),
$$


with $\xi$ the fixed permutation $(12)(34) \ldots(2 n-1,2 n)$. With the same notation, the normal ordered 2pt-function writes

$$
\left\langle O_{\sigma_{1}, \sigma_{2}, \sigma_{3}} O_{\tau_{1}, \tau_{2}, \tau_{3}}\right\rangle=\sum_{\mu \in S_{2 n}} N^{\sum_{i=1}^{3} \mathbf{c}\left(\mu^{-1} \widetilde{\tau}_{i} \mu \widetilde{\sigma}_{i}\right)}
$$

Aiming at finding an orthogonal base of 2pt-functions, the central elements $\Omega_{i}=\sum_{\alpha_{i} \in S_{2 n}} N^{\mathrm{c}\left(\alpha_{i}\right)-2 n} \alpha_{i}$ will be of great use. We have

$$
\begin{aligned}
& \left\langle O_{\sigma_{1}, \sigma_{2}, \sigma_{3}} O_{\tau_{1}, \tau_{2}, \tau_{3}}\right\rangle=N^{6 n} \sum_{\mu} \delta_{3}\left[\left(\mu^{-1}\right)^{\otimes 3}\left(\widetilde{\tau}_{1} \otimes \widetilde{\tau}_{2} \otimes \widetilde{\tau}_{3}\right) \mu^{\otimes 3}\left(\widetilde{\sigma}_{1} \otimes \widetilde{\sigma}_{2} \otimes \widetilde{\sigma}_{3}\right)\left(\Omega_{1} \otimes \Omega_{2} \otimes \Omega_{3}\right)\right] \\
& =N^{6 n} \sum_{\mu} \delta_{3}\left[\left(\widetilde{\tau}_{1} \otimes \widetilde{\tau}_{2} \otimes \widetilde{\tau}_{3}\right) \mu^{\otimes 3}\left(\widetilde{\sigma}_{1} \otimes \widetilde{\sigma}_{2} \otimes \widetilde{\sigma}_{3}\right)\left(\mu^{-1}\right)^{\otimes 3}\left(\Omega_{1} \otimes \Omega_{2} \otimes \Omega_{3}\right)\right] .
\end{aligned}
$$

We then introduce the representation theoretic elements $O^{R_{1}, R_{2}, R_{3}, \tau}$ as

$$
O^{R_{1}, R_{2}, R_{3}, \tau}=\sum_{\sigma_{l}} \delta_{3}\left(Q^{R_{1}, R_{2}, R_{3}, \tau} \sigma_{1}^{-1} \otimes \sigma_{2}^{-1} \otimes \sigma_{3}^{-1}\right) O_{\sigma_{1}, \sigma_{2}, \sigma_{3}}
$$

and, after a lengthy calculation, we arrive at

$$
\begin{aligned}
& \left\langle O^{R_{1}, R_{2}, R_{3}, \tau} O^{R_{1}^{\prime}, R_{2}^{\prime}, R_{3}^{\prime}, \tau^{\prime}}\right\rangle=\left[\prod_{i=1}^{3} \delta_{R_{i}^{\prime} R_{i}}\right] \delta_{\tau^{\prime} \tau} F\left(R_{1}, R_{2}, R_{3}, \tau\right) \\
& F\left(R_{1}, R_{2}, R_{3}, \tau\right)=\sum_{S_{i}, \tau_{i}}\left[\prod_{i=1}^{3} \operatorname{Dim}_{N}\left(S_{i}\right)\right]\left[\sum_{b_{i}, c_{i}, p_{i}} D_{b_{i} c_{i}}^{S_{i}}(\xi) C_{b_{i}, c_{i} ; p_{i}}^{S_{i}, S_{i} ; \tau_{i}, \tau_{i}} B_{p_{i}}^{R_{i}, t r}\right]^{2} .
\end{aligned}
$$

This is precisely the orthogonality of the representation theoretic base $\left\{O^{R_{1}, R_{2}, R_{3}, \tau}\right\}$ for normal ordered Gaussian correlators in $\mathscr{K}_{3}^{o}(2 n)$.

\section{Conclusion}

We have enumerated rank $d$ real and complex tensor invariants that are orthogonal and unitary invariants relying on techniques build on symmetric groups and their representation theory. From this enumeration, we find several bridges with other formalisms. In particular, we were interested in TopFT allowing us to interpret in a different way these countings as the number of covers of topological objects (punctured 2-sphere for the complex case and torus with defects for the real case) and even to foresee geometrical pictures attached to them. If this program is successfully achieved then one could establish, for instance, connections between string theory and tensor models. Such correspondences deserve attention. From another side, the representation theory of the symmetric group brings a different perspective on these countings and might connect them with the problem of the combinatorial interpretation of the Kronecker coefficient. This could be of major interest in computational complexity theory. Moreover, we have found that tensor model observables span a graph algebra with interesting properties such as associativity and semi-simplicity. With the latter feature, the complex case exhibits a Wedderburn-Artin base. Finally, we were interested in Gaussian correlators, showing that there are representation theoretic orthogonal bases for the normal ordered 2pt-function. Aiming at extracting physical properties, more work must be performed on computable sectors in tensor models. With the help of computer softwares, the fast calculation of the $1 \mathrm{pt}$-function is encouraging. Higher order correlators need to be addressed after this.

The following table delivers a summary of the results presented in this work. 


\begin{tabular}{|c||c|c|}
\hline & Unitary TM & Orthogonal TM \\
\hline Counting observables $d$ & & \\
\# of observables at $d=3$ & $\sqrt{ }$ & $\sqrt{ }$ \\
TopFT 2 interpretation & Branched covers of the 2-sphere & Covers of 2-torus with defects \\
Algebraic structure & associative unital semi-simple & associative unitary semi-simple \\
Invariant ortho. rep. base & $\sqrt{ }$ & $\sqrt{ }$ \\
1-pt and 2-pt correlators & $\sqrt{ }$ & $\sqrt{ }$ \\
Wedderburn-Artin base & $\sqrt{ }$ & \\
\hline
\end{tabular}

Finally, we may venture a few words about new tensor models mixing both $U(N)$ and $O(N)$ invariance. Inspired by the work of Ferrari [86], a tensor model with mixed invariance under $U(N)^{\times 2} \times O(N)$ has been recently studied by Benedetti et al [87]. Note that this type of mixed invariance was first introduced in the so-called multi-orientable tensor model [44]. Both these mixed-type models admit a large $N$ expansion and have interesting scaling properties. We can certainly apply the above counting formalism to such models promoting a mixed group $U(N)^{\times p} \times$ $O(N)^{\times q}$. For simplicity, consider the case of a rigid tensor (no symmetry under the tensor index), of rank $d=p+q$, require that the number of $T$ in the observable is even (same for the number of $\bar{T}$ ), and that no indices associated with an orthogonal invariance contract a $T$ and a $\bar{T}$ (apart from the quadratic invariant $\operatorname{Tr}_{2}(T \bar{T})$ ). The counting operates with three groups of permutation $\left(\sigma_{1}, \sigma_{2}, \ldots, \sigma_{p}\right) \in S_{2 n}^{\times p}$ implementing the connection between $T$ 's and $\bar{T}$ 's, $\left(\tau_{1}, \tau_{2}, \ldots, \tau_{q}\right) \in S_{2 n}^{\times q}$ establishing the connection between the $T$ 's only, and $\left(\bar{\tau}_{1}, \bar{\tau}_{2}, \ldots, \bar{\tau}_{q}\right) \in S_{2 n}^{\times q}$ connecting the $\bar{T}$ 's between themselves. The invariants are fully determined by the equivalence

$$
\begin{aligned}
& {\left[\tau_{1}, \tau_{2}, \ldots, \tau_{q} ; \sigma_{1}, \sigma_{2}, \ldots, \sigma_{p} ; \bar{\tau}_{1}, \bar{\tau}_{2}, \ldots, \bar{\tau}_{q}\right]} \\
& \quad \sim\left[\gamma_{1} \tau_{1} \mu_{1}, \gamma_{2} \tau_{2} \mu_{1}, \ldots, \gamma_{q} \tau_{q} \mu_{1} ; \mu_{1} \sigma_{1} \mu_{2}, \mu_{1} \sigma_{2} \mu_{2}, \ldots, \mu_{1} \sigma_{p} \mu_{2} ; \mu_{2} \bar{\tau}_{1} \bar{\gamma}_{1}, \mu_{2} \bar{\tau}_{2} \bar{\gamma}_{2}, \ldots, \mu_{2} \bar{\tau}_{q} \bar{\gamma}_{q}\right]
\end{aligned}
$$

where $\gamma_{i}$ and $\bar{\gamma}_{i} \in S_{2 n}\left[S_{2}\right]$, and $\mu_{i} \in S_{2 n}$. Note that this is no longer a pure left and then right action as in the cases treated above. As preliminary thoughts, sorting these classes may require to embed it in tensor product spaces: $\mathbb{C}\left(S_{2 n}^{\times q}\right) \otimes \mathbb{C}\left(S_{2 n}^{\times p}\right) \otimes \mathbb{C}\left(S_{2 n}^{\times q}\right)$. Then define, four actions: $S_{2 n}\left[S_{2}\right]^{\times q} \otimes 1 \otimes 1$ that acts on the left and only on the first slot; $\operatorname{Diag}_{q \otimes p}^{R, L}\left(S_{2 n}\right) \otimes 1$ acts by diagonal (right, left) multiplication: the right multiplication acts on the $q \tau_{i}$ 's on the first slot, and the left multiplication on the next $p \sigma_{i}$ 's factors on the second slot; next we have $1 \otimes \operatorname{Diag}_{p \otimes q}^{R, L}\left(S_{2 n}\right)$, that acts similarly (note the exchange of roles of $p$ and $q$ ) but on the second and third slots; finally $1 \otimes 1 \otimes S_{2 n}\left[S_{2}\right]^{\times q}$ acting on the right and only on the third slot:

$$
\begin{aligned}
& {\left[S_{2 n}\left[S_{2}\right]^{\times q} \otimes 1 \otimes 1\right]\left[\operatorname{Diag}_{q \otimes p}^{R, L}\left(S_{2 n}\right) \otimes 1\right]\left[1 \otimes \operatorname{Diag}_{p \otimes q}^{R, L}\left(S_{2 n}\right)\right]\left[1 \otimes 1 \otimes S_{2 n}\left[S_{2}\right]^{\times q}\right]} \\
& \triangleright \mathbb{C}\left(S_{2 n}^{\times q}\right) \otimes \mathbb{C}\left(S_{2 n}^{\times p}\right) \otimes \mathbb{C}\left(S_{2 n}^{\times q}\right) .
\end{aligned}
$$

The fact that $\operatorname{Diag}_{q \otimes p}^{R, L}\left(S_{2 n}\right) \otimes 1$ and $1 \otimes \operatorname{Diag}_{p \otimes q}^{R, L}\left(S_{2 n}\right)$ do not form groups make this new puzzle interesting. 


\section{Acknowledgments}

I gratefully thanks the organisers of Corfu Summer Institute 2019 "School and Workshops on Elementary Particle Physics and Gravity" (CORFU2019) and the Humboldt Kolleg "Frontiers in Physics, From the Electroweak to the Planck Scales", especially George Zoupanos, Patrizia Vitale and Ifigeneia Moraiti. I am also particularly indebted to Sanjaye Ramgoolam for introducing me in the subject and for years of collaboration without which this work could not stand. I wish also to warmly thank Avohou Remi Cocou and Nicolas Dub, for collaboration on the joint work presented here.

\section{References}

[1] J. Ambjorn, B. Durhuus and T. Jonsson, "Three-Dimensional Simplicial Quantum Gravity And Generalized Matrix Models,” Mod. Phys. Lett. A 6, 1133 (1991).

[2] M. Gross, "Tensor models and simplicial quantum gravity in > 2-D," Nucl. Phys. Proc. Suppl. 25A, 144 (1992).

[3] N. Sasakura, "Tensor model for gravity and orientability of manifold," Mod. Phys. Lett. A 6, 2613 (1991).

[4] P. Di Francesco, P. H. Ginsparg and J. Zinn-Justin, "2-D Gravity and random matrices," Phys. Rept. 254, 1 (1995) [arXiv:hep-th/9306153].

[5] R. Gurau, "Random Tensors," Oxford University Press, Oxford, 2016.

[6] S. Carrozza and V. Pozsgay, "SYK-like tensor quantum mechanics with $\operatorname{Sp}(N)$ symmetry," Nucl. Phys. B 941, 28 (2019) [arXiv:1809.07753 [hep-th]].

[7] R. C. Avohou, J. Ben Geloun and N. Dub, "On the counting of $O(N)$ tensor invariants," accepted in Advances in Theoretical and Mathematical Physics (2020), arXiv:1907.04668 [math-ph].

[8] J. Ben Geloun and S. Ramgoolam, "Counting tensor model observables and branched covers of the 2-sphere," Ann. Inst. Henri Poincaré D 1 (2014), 77-138, arXiv:1307.6490.

[9] P. Diaz and S. J. Rey, “Orthogonal Bases of Invariants in Tensor Models," JHEP 1802, 089 (2018) [arXiv:1706.02667 [hep-th]].

[10] R. de Mello Koch, D. Gossman and L. Tribelhorn, "Gauge Invariants, Correlators and Holography in Bosonic and Fermionic Tensor Models," JHEP 1709, 011 (2017) [arXiv:1707.01455 [hep-th]].

[11] J. Ben Geloun and S. Ramgoolam, "Tensor Models, Kronecker coefficients and Permutation Centralizer Algebras," JHEP 1711, 092 (2017) [arXiv:1708.03524 [hep-th]].

[12] H. Itoyama, A. Mironov and A. Morozov, "Cut and join operator ring in tensor models," Nucl. Phys. B 932, 52 (2018) [arXiv:1710.10027 [hep-th]].

[13] P. Diaz and S. J. Rey, "Invariant Operators, Orthogonal Bases and Correlators in General Tensor Models,” Nucl. Phys. B 932, 254 (2018) [arXiv:1801.10506 [hep-th]].

[14] P. Diaz, "Tensor and Matrix models: a one-night stand or a lifetime romance?," JHEP 1806, 140 (2018) [arXiv:1803.04471 [hep-th]].

[15] H. Itoyama, A. Mironov and A. Morozov, "From Kronecker to tableau pseudo-characters in tensor models," Phys. Lett. B 788, 76 (2019) [arXiv:1808.07783 [hep-th]]. 
[16] H. Itoyama and R. Yoshioka, "Generalized cut operation associated with higher order variation in tensor models,” Nucl. Phys. B 945, 114681 (2019) [arXiv:1903.10276 [hep-th]].

[17] H. Itoyama, A. Mironov and A. Morozov, “Tensorial generalization of characters," JHEP 1912, 127 (2019) doi:10.1007/JHEP12(2019)127 [arXiv:1909.06921 [hep-th]].

[18] N. Amburg, H. Itoyama, A. Mironov, A. Morozov, D. Vasiliev and R. Yoshioka, "Correspondence between Feynman diagrams and operators in quantum field theory that emerges from tensor model," arXiv:1911.10574 [hep-th].

[19] S. Corley, A. Jevicki and S. Ramgoolam, "Exact correlators of giant gravitons from dual N=4 SYM theory," Adv. Theor. Math. Phys. 5 (2002) 809 [hep-th/0111222].

[20] S. Corley and S. Ramgoolam, "Finite factorization equations and sum rules for BPS correlators in N=4 SYM theory,” Nucl. Phys. B 641 (2002) 131 [hep-th/0205221].

[21] T. W. Brown, P. J. Heslop and S. Ramgoolam, "Diagonal multi-matrix correlators and BPS operators in N=4 SYM," JHEP 0802 (2008) 030 [arXiv:0711.0176 [hep-th]].

[22] G. Kemp and S. Ramgoolam, "BPS states, conserved charges and centres of symmetric group algebras,” JHEP 01, 146 (2020) [arXiv:1911.11649 [hep-th]].

[23] S. Ramgoolam, "Schur-Weyl duality as an instrument of Gauge-String duality," AIP Conf. Proc. 1031, no.1, 255-265 (2008) [arXiv:0804.2764 [hep-th]].

[24] R. de Mello Koch and S. Ramgoolam, "From Matrix Models and Quantum Fields to Hurwitz Space and the absolute Galois Group," arXiv:1002.1634 [hep-th].

[25] R. de Mello Koch and S. Ramgoolam, "Strings from Feynman Graph counting : without large N," Phys. Rev. D 85, 026007 (2012) doi:10.1103/PhysRevD.85.026007 [arXiv:1110.4858 [hep-th]].

[26] R. de Mello Koch and S. Ramgoolam, “A double coset ansatz for integrability in AdS/CFT,” JHEP 1206, 083 (2012) doi:10.1007/JHEP06(2012)083 [arXiv:1204.2153 [hep-th]].

[27] R. de Mello Koch, S. Ramgoolam and C. Wen, "On the refined counting of graphs on surfaces," Nucl. Phys. B 870 (2013) 530 [arXiv:1209.0334 [hep-th]].

[28] J. Pasukonis and S. Ramgoolam, “Quivers as Calculators: Counting, Correlators and Riemann Surfaces,” JHEP 1304, 094 (2013) [arXiv:1301.1980 [hep-th]].

[29] P. Caputa, R. de Mello Koch and P. Diaz, "A basis for large operators in N=4 SYM with orthogonal gauge group,” JHEP 1303, 041 (2013) [arXiv:1301.1560 [hep-th]].

[30] P. Mattioli and S. Ramgoolam, "Permutation Centralizer Algebras and Multi-Matrix Invariants," Phys. Rev. D 93, no. 6, 065040 (2016) [arXiv:1601.06086 [hep-th]].

[31] S. Ramgoolam and M. Sedlák, "Quantum Information Processing and Composite Quantum Fields," JHEP 01, 170 (2019) [arXiv:1809.05156 [hep-th]].

[32] D. Kartsaklis, S. Ramgoolam and M. Sadrzadeh, "Linguistic Matrix Theory,” [arXiv:1703.10252 [cs.CL]].

[33] S. Ramgoolam, "Permutation invariant Gaussian matrix models," Nucl. Phys. B 945, 114682 (2019) [arXiv:1809.07559 [hep-th]].

[34] V. Rivasseau, “The Tensor Track, IV,” PoS CORFU2015, 106 (2016) [arXiv:1604.07860 [hep-th]].

[35] V. Rivasseau, “The Tensor Track, III,” Fortsch. Phys. 62, 81-107 (2014) [arXiv:1311.1461 [hep-th]]. 
[36] N. Delporte and V. Rivasseau, "The Tensor Track V: Holographic Tensors," [arXiv:1804.11101 [hep-th]].

[37] R. Gurau, “Colored Group Field Theory,” Commun. Math. Phys. 304, 69 (2011) [arXiv:0907.2582 [hep-th]].

[38] R. Gurau, "The complete $1 / \mathrm{N}$ expansion of colored tensor models in arbitrary dimension," Annales Henri Poincare 13, 399 (2012) [arXiv:1102.5759 [gr-qc]].

[39] G. 't Hooft, “A Planar Diagram Theory for Strong Interactions,” Nucl. Phys. B 72, 461 (1974).

[40] V. Bonzom, R. Gurau, A. Riello and V. Rivasseau, "Critical behavior of colored tensor models in the large N limit,” Nucl. Phys. B 853, 174 (2011) [arXiv:1105.3122 [hep-th]].

[41] R. Gurau and J. P. Ryan, "Melons are branched polymers," Annales Henri Poincare 15, no. 11, 2085 (2014) [arXiv:1302.4386 [math-ph]].

[42] R. Gurau, "Universality for Random Tensors," Ann. Inst. H. Poincare Probab. Statist. 50, no. 4, 1474 (2014) [arXiv:1111.0519 [math.PR]].

[43] S. Carrozza and A. Tanasa, " $O(N)$ Random Tensor Models," Lett. Math. Phys. 106, no. 11, 1531 (2016) [arXiv:1512.06718 [math-ph]].

[44] A. Tanasa, "The Multi-Orientable Random Tensor Model, a Review," SIGMA 12, 056 (2016) [arXiv:1512.02087 [hep-th]].

[45] J. Ben Geloun and V. Rivasseau, "A Renormalizable 4-Dimensional Tensor Field Theory," Commun. Math. Phys. 318, 69 (2013) [arXiv:1111.4997 [hep-th]].

[46] J. Ben Geloun and D. O. Samary, "3D Tensor Field Theory: Renormalization and One-loop $\beta$-functions," Annales Henri Poincare 14, 1599 (2013) [arXiv:1201.0176 [hep-th]].

[47] J. Ben Geloun, "Renormalizable Models in Rank $d \geq 2$ Tensorial Group Field Theory," Commun. Math. Phys. 332, 117 (2014) [arXiv:1306.1201 [hep-th]].

[48] S. Carrozza, "Tensorial methods and renormalization in Group Field Theories," Springer Theses, 2014 (Springer, NY, 2014), arXiv:1310.3736 [hep-th].

[49] D. O. Samary and F. Vignes-Tourneret, "Just Renormalizable TGFT's on $U(1)^{d}$ with Gauge Invariance," Communications in Mathematical Physics (2014) [arXiv:1211.2618 [hep-th]].

[50] D. O. Samary, "Closed equations of the two-point functions for tensorial group field theory," Class. Quant. Grav. 31, 185005 (2014) [arXiv:1401.2096 [hep-th]].

[51] C. I. Pérez-Sánchez, "The full Ward-Takahashi Identity for colored tensor models," Commun. Math. Phys. 358, no. 2, 589 (2018) [arXiv:1608.08134 [math-ph]].

[52] T. Krajewski, V. Rivasseau and V. Sazonov, "Constructive Matrix Theory for Higher Order Interaction II: Hermitian and Real Symmetric Cases," [arXiv:1910.13261 [math-ph]].

[53] J. Ben Geloun and R. Toriumi, "Renormalizable enhanced tensor field theory: The quartic melonic case,” J. Math. Phys. 59, no. 11, 112303 (2018) [arXiv:1709.05141 [hep-th]].

[54] A. Eichhorn and T. Koslowski, "Continuum limit in matrix models for quantum gravity from the Functional Renormalization Group,” Phys. Rev. D 88, 084016 (2013) [arXiv:1309.1690 [gr-qc]].

[55] A. Eichhorn and T. Koslowski, "Flowing to the continuum in discrete tensor models for quantum gravity,” Ann. Inst. H. Poincare Comb. Phys. Interact. 5, no. 2, 173 (2018) [arXiv:1701.03029 [gr-qc]]. 
[56] D. Benedetti, J. Ben Geloun and D. Oriti, "Functional Renormalisation Group Approach for Tensorial Group Field Theory: a Rank-3 Model,” JHEP 1503, 084 (2015) [arXiv:1411.3180 [hep-th]].

[57] J. Ben Geloun, R. Martini and D. Oriti, "Functional Renormalization Group analysis of a Tensorial Group Field Theory on $\mathbb{R}^{3}$," EPL 112, no. 3, 31001 (2015) [arXiv:1508.01855 [hep-th]].

[58] J. Ben Geloun, R. Martini and D. Oriti, "Functional Renormalisation Group analysis of Tensorial Group Field Theories on $\mathbb{R}^{d}$," Phys. Rev. D 94, no. 2, 024017 (2016) [arXiv:1601.08211 [hep-th]].

[59] A. Eichhorn, T. Koslowski, J. Lumma and A. D. Pereira, "Towards background independent quantum gravity with tensor models," arXiv:1811.00814 [gr-qc].

[60] A. Eichhorn, J. Lumma, A. D. Pereira and A. Sikandar, "Universal critical behavior in tensor models for four-dimensional quantum gravity," JHEP 02, 110 (2020) [arXiv:1912.05314 [gr-qc]].

[61] V. Lahoche and D. Ousmane Samary, "Nonperturbative renormalization group beyond the melonic sector: The effective vertex expansion method for group fields theories," Phys. Rev. D 98, no. 12, 126010 (2018) [arXiv:1809.00247 [hep-th]].

[62] V. Lahoche and D. Ousmane Samary, "Unitary symmetry constraints on tensorial group field theory renormalization group flow," Class. Quant. Grav. 35, no. 19, 195006 (2018) [arXiv:1803.09902 [hep-th]].

[63] V. Lahoche and D. O. Samary, "Ward-constrained melonic renormalization group flow," arXiv:1904.05655 [hep-th].

[64] A. Kitaev, "A simple model of quantum holography," Talks at KITP, April 7, 2015 and May 27, 2015, http://online.kitp.ucsb.edu/online/entangled15/kitaev/.

[65] J. Maldacena and D. Stanford, "Remarks on the Sachdev-Ye-Kitaev model," Phys. Rev. D 94106002 (2016) [arXiv:1604.07818 [hep-th]].

[66] E. Witten, "An SYK-Like Model Without Disorder," arXiv:1610.09758 [hep-th]; R. Gurau, "The complete 1/ $N$ expansion of a SYK-like tensor model," Nucl. Phys. B 916, 386 (2017) [arXiv:1611.04032 [hep-th]].

[67] J. Ben Geloun and V. Rivasseau, "A Renormalizable SYK-type Tensor Field Theory,” Annales Henri Poincare 19, no.11, 3357-3395 (2018) [arXiv:1711.05967 [hep-th]].

[68] D. Benedetti, R. Gurau and S. Harribey, "Line of fixed points in a bosonic tensor model," JHEP 06, 053 (2019) [arXiv:1903.03578 [hep-th]].

[69] D. Benedetti, N. Delporte, S. Harribey and R. Sinha, "Sextic tensor field theories in rank 3 and 5," [arXiv:1912.06641 [hep-th]].

[70] D. Benedetti, R. Gurau, S. Harribey and K. Suzuki, "Hints of unitarity at large $N$ in the $O(N)^{3}$ tensor field theory," JHEP 02, 072 (2020) [arXiv:1909.07767 [hep-th]].

[71] D. Benedetti, "Melonic CFTs," [arXiv:2004.08616 [hep-th]].

[72] N. Delporte and V. Rivasseau, "The Tensor Track VI: Field Theory on Random Trees and SYK on Random Unicyclic Graphs," [arXiv:2004.13744 [hep-th]].

[73] The On-Line Encyclopedia of Integer Sequences, http://oeis.org.

[74] Schneps, Leila, ed. (1994), The Grothendieck Theory of Dessins d'Enfants, London Mathematical Society Lecture Note Series, Cambridge: Cambridge University Press, ISBN 978-0-521-47821-2. 
[75] M. Hammermesh, "Group Theory and its Application to Physical Problems," Addison-Wesley, Massachusetts, 1962.

[76] R. Stanley, "Positivity problems and conjectures in Algebraic Combinatorics," September 1999, http://www-math.mit.edu/rstan/papers/problems.pdf .

[77] C. Ikenmeyer, K. D. Mulmuley, and M. Walter, "On vanishing of Kronecker coefficients," arXiv:1507.02955[cs.CC].

[78] J. Blasiak, "Kronecker coefficients for one hook shape," arXiv:1209.2018 [math.CO].

[79] R.C. Read, "The enumeration of locally restricted graphs," Journal London Math.Soc. 34 (1959), 417-436.

[80] S. Cordes, G. W. Moore and S. Ramgoolam, "Large N 2-D Yang-Mills theory and topological string theory," Commun. Math. Phys. 185, 543 (1997) [hep-th/9402107].

[81] S. Cordes, G. W. Moore and S. Ramgoolam, "Lectures on 2-d Yang-Mills theory, equivariant cohomology and topological field theories," Nucl. Phys. Proc. Suppl. 41, 184 (1995) [hep-th/9411210].

[82] R. Howe, "Perspectives on invariant theory:Schur duality, multiplicity-free actions and beyond," in: The Schur Lectures, 1999, Israel Mathematical Conference Proceedings, Vol. 8 (1995), pp. 1-182.

[83] H. Mizukawa, "Wreath product generalizartion of the triple $\left(S_{n}, H_{n}, \varphi\right)$ and their spherical functions," Journal of Algebra 334 31-53 (2011), arXiv:0908.3056 [math.RT].

[84] I. G. Macdonald, "Symmetric Functions and Hall Polynomials," 2nd ed., Oxford Univ. Press (1995).

[85] V. N. Ivanov, "Bispherical functions on the symmetric group associated with the hyperoctahedral subgroup," Jour. of Math. Sciences 96, 3505 (1999).

[86] F. Ferrari, “The Large D Limit of Planar Diagrams," [arXiv:1701.01171 [hep-th]].

[87] D. Benedetti, S. Carrozza, R. Toriumi and G. Valette, "Multiple scaling limits of $\mathrm{U}(N)^{2} \times \mathrm{O}(D)$ multi-matrix models," [arXiv:2003.02100 [math-ph]]. 